\title{
Arrangement of Excitatory Synaptic Inputs on Dendrites of the Medial Superior Olive
}

\author{
Alexander R. Callan, ${ }^{1,2}$ Martin Heß, ${ }^{1}$ Felix Felmy, ${ }^{3 *}$ and ${ }^{\oplus}$ Christian Leibold ${ }^{1,4 *}$ \\ ${ }^{1}$ Department Biology II, Ludwig-Maximilians-Universität München, München, Germany, 82152, ${ }^{2}$ Graduate School of Systemic Neurosciences, \\ Ludwig-Maximilians-Universität München, München, Germany, $82152,{ }^{3}$ Zoologisches Institut, Stiftung Tierärztliche Hochschule Hannover, \\ Hannover, Germany, 30559, and ${ }^{4}$ Bernstein Center for Computational Neuroscience, München, Germany, 82152
}

Neurons in the medial superior olive (MSO) detect $10 \mu$ s differences in the arrival times of a sound at the two ears. Such acuity requires exquisitely precise integration of binaural synaptic inputs. There is substantial understanding of how neuronal phase locking of afferent MSO structures, and MSO membrane biophysics subserve such high precision. However, we still lack insight into how the entirety of excitatory inputs is integrated along the MSO dendrite under sound stimulation. To understand how the dendrite integrates excitatory inputs as a whole, we combined anatomic quantifications of the afferent innervation in gerbils of both sexes with computational modeling of a single cell. We present anatomic data from confocal and transmission electron microscopy showing that single afferent fibers follow a single dendrite mostly up to the soma and contact it at multiple (median 4) synaptic sites, each containing multiple independent active zones (the overall density of active zones is estimated as 1.375 per $\mu \mathrm{m}^{2}$ ). Thus, any presynaptic action potential may elicit temporally highly coordinated synaptic vesicle release at tens of active zones, thereby achieving secure transmission. Computer simulations suggest that such an anatomic arrangement boosts the amplitude and sharpens the time course of excitatory postsynaptic potentials by reducing current sinks and more efficiently recruiting subthreshold potassium channels. Both effects improve binaural coincidence detection compared with single large synapses at the soma. Our anatomic data further allow for estimation of a lower bound of 7 and an upper bound of 70 excitatory fibers per dendrite.

Key words: binaural hearing; coincidence detection; dendritic computation; innervation pattern; ITD; MSO

Significance Statement

Passive dendritic propagation attenuates the amplitude of postsynaptic potentials and widens their temporal spread. Neurons in the medial superior olive, with their large bilateral dendrites, however, can detect coincidence of binaural auditory inputs with submillisecond precision, a computation that is in stark contrast to passive dendritic processing. Here, we show that dendrites can counteract amplitude attenuation and even decrease the temporal spread of postsynaptic potentials, if active subthreshold potassium conductances are triggered in temporal coordination along the whole dendrite. Our anatomic finding that axons run in parallel to the dendrites and make multiple synaptic contacts support such coordination since incoming action potentials would depolarize the dendrite at multiple sites within a brief time interval.

\section{Introduction}

To pinpoint the source of a low-frequency sound, humans rely on interaural time differences (ITDs), the differences between the arrival times of sounds at the two ears (Grothe et al., 2010),

\footnotetext{
Received May 4, 2020; revised Nov. 5, 2020; accepted Nov. 10, 2020.

Author contributions: A.R.C., F.F., and C.L. designed research; A.R.C. and M.H. performed research; A.R.C. and M.H. analyzed data; A.R.C. wrote the first draft of the paper; A.R.C., M.H., F.F., and C.L. edited the paper; F.F. and C.L. wrote the paper.

*F.F. and C.L. contributed equally to this work.

This work was supported by Deutsche Forschungsgemeinschaft Grant CRC870. We thank Benedikt Grothe and Olga Alexandrova for support.

The authors declare no competing financial interests.

Correspondence should be addressed to Christian Leibold at leibold@bio.Imu.de.

https://doi.org/10.1523/JNEUROSCl.1055-20.2020

Copyright $\odot 2021$ the authors
}

and can detect differences in ITDs as low as $10 \mu$ s (Klumpp and Eady, 1956). The medial superior olive (MSO) is the first neuronal stage where phase-locked bilateral auditory inputs are combined, and its neurons encode variations in ITD with submillisecond resolution (Goldberg and Brown, 1969; Yin and Chan, 1990).

The dendritic trees of MSO neurons typically exhibit a bipolar morphology, with two main dendrites emerging from opposite sides of the cell soma (Stotler, 1953; Rautenberg et al., 2009). Each MSO neuron receives excitatory glutamatergic and inhibitory glycinergic input (Brand et al., 2002; Pecka et al., 2008; Myoga et al., 2014). Inhibitory, phase-locked inputs (Franken et al., 2015) originate from the lateral and medial nuclei of the trapezoid body, and become confined to the soma and proximal dendrites during development (Clark, 1969; Kapfer et al., 2002; 
Werthat et al., 2008; Couchman et al., 2012). Excitatory inputs originate from bushy cells in the cochlear nucleus. These inputs are segregated by side, with the lateral dendrite receiving inputs from the ipsilateral cochlear nucleus, and the medial dendrite receiving inputs from the contralateral cochlear nucleus (Stotler, 1953). ITD computation in MSO neurons strongly depends on detecting the coincidence of these excitatory inputs to each dendrite, even in the presence of phase-locked inhibition (Leibold, 2010).

Because of the extreme temporal resolution of ITD processing, even minor temporal distortions at any stage within the circuitry will functionally impact neuronal ITD detection. This includes distortions because of the cellular structure of MSO neurons themselves (Zhou et al., 2005). Moreover, the location of a synaptic input on the dendrite will affect the timing and kinetics of the EPSPs. In passive dendrites, EPSP amplitudes are attenuated as they propagate toward the soma, and dendritic filtering causes their half-widths to increase, thereby shifting the peak latency. In MSO neurons, dendritic filtering has been shown to be counteracted by a gradient of EPSP sizes (Winters et al., 2017) and an active sharpening because of low threshold potassium channels (Mathews et al., 2010). Despite these adaptations, MSO neurons must nevertheless contend with delays in propagation along the dendrite (Winters et al., 2017), since bilateral stimulation of an MSO neuron by axons that terminate at different distances from the soma on their respective dendrites would introduce an additional binaural delay. The impact of such EPSP propagation delay would likely depend on the pattern of axonal innervation of MSO dendrites. Previous axonal tracing studies have shown axonal branching patterns of cochlear nucleus neurons in the MSO but have not related these axonal branching patterns to innervation of individual MSO neurons (Smith et al., 1993; Beckius et al., 1999).

A further source of temporal jitter arises from the number of input fibers. Previously, it was found that only few excitatory fibers are necessary to evoke MSO action potentials at rest (Couchman et al., 2010); however, the absolute number of input fibers is still not known. Few strong independent release sites, however, would introduce large Poisson noise into the dendritic integration process; thus, an in-depth investigation of the spatial arrangement and number of release sites of single fibers is necessary to assess such noise contributions.

Here, we investigated the pattern of excitatory innervation of single MSO neurons, and the spatial arrangement of active zones, a proxy for release sites, within boutons. We show that individual axons typically form multiple synaptic contact sites along the dendrites of MSO neurons. Most axons therefore innervate a length of dendrite rather than a specific point. Boutons typically contain multiple active zones, which presumably reduce synaptic noise. Transmission electron microscopy (TEM) data suggest the absence of myelin close to the contact sites. Computational modeling indicates that innervation by distributed synapses enhances ITD sensitivity in MSO neurons.

\section{Materials and Methods}

Animals. All experiments were conducted with tissue taken from male and female Mongolian gerbils (Meriones unguiculatus). All experiments were conducted according to institutional guidelines, and regional and national laws. Before experiments, animals were housed with littermates and nonlittermates of the same sex and age in temperature-controlled rooms with a $12 \mathrm{~h}$ light-dark cycle.

Axon filling and immunohistochemistry. Axon filling with biocytin was performed following Seidl and Rubel (2016). Male and female
Table 1. Primary antibodies

\begin{tabular}{llllll}
\hline Antigen & Host & Type & Dilution & Company & Catalog \# \\
\hline MAP2 & Chicken & Polyclonal IgY & $1: 500$ & Acris & TA336617 \\
VGluT1 & Guinea pig & Polyclonal & $1: 2000$ & Synaptic Systems & 135304 \\
GlyT2 & Rabbit & Polyclonal & $1: 1000$ & Synaptic Systems & 272003 \\
\hline
\end{tabular}

Table 2. Secondary antibodies

\begin{tabular}{llllll}
\hline Antigen & Conjugate & Host & Dilution & Company & Catalog \# \\
\hline Chicken & Cy3 & Donkey & $1: 200$ & Dianova & $703-166-155$ \\
Guinea pig & Alexa-647 & Donkey & $1: 100$ & Dianova & $706-605-148$ \\
Rabbit & AMCA & Donkey & $1: 100$ & Dianova & $711-155-152$ \\
\hline
\end{tabular}

gerbils aged P21 to P30 were used for axon tracing and immunohistochemistry. Animals were anesthetized with isoflurane (Zoetis), and brains were quickly removed in ice-cold ACSF. ACSF contained the following (in mM): $125 \mathrm{NaCl}, 25 \mathrm{NaHCO}_{3}, 1.25 \mathrm{NaH}_{2} \mathrm{PO}_{4}, 2.5 \mathrm{KCl}, 25$ glucose, 0.4 ascorbic acid, 3 myo-inositol, $2 \mathrm{Na}$-pyruvate, $1 \mathrm{MgCl}_{2}$, and 2 $\mathrm{CaCl}_{2}$. Brains were affixed to a cold Sylgard-based dish by pins through the cerebrum, and submerged in ice-cold ACSF. Brains were positioned so that one cochlear nucleus faced upwards. Under a dissecting microscope, a crystal of biocytin was placed directly on the cochlear nucleus. The brain was then moved to an incubation chamber containing ACSF bubbled with carbogen, and left to incubate at room temperature for 2$2.5 \mathrm{~h}$.

Following incubation, brains were immediately submerged in $4 \%$ PFA in $0.02 \mathrm{M}$ PBS for 12-14 h. Brains were subsequently washed 3 times for at least $10 \mathrm{~min}$ in PBS. Slices of thickness 120 or $160 \mu \mathrm{m}$ were made with a VT1200S Vibratome (Leica Microsystems).

Free-floating slices were incubated in blocking solution containing $0.1 \% \mathrm{v} / \mathrm{v}$ saponin, $1 \% \mathrm{w} / \mathrm{v}$ BSA, and $1 \%$ or $1.5 \% \mathrm{v} / \mathrm{v}$ Triton X-100 in $0.02 \%$ PBS. Subsequent to blocking, slices were incubated in AlexaFluor488-conjugated streptavidin (Sigma Millipore) at 1:500 dilution along with primary antibodies for 46-72 h. For details of all primary antibodies used, see Table 1 . All primary and secondary antibodies were diluted in blocking solution. Slices were subsequently washed 3 times for $30 \mathrm{~min}$ each time in blocking solution, then incubated for $48-72 \mathrm{~h}$ in fluorophore-conjugated secondary antibodies. For the list of all secondary antibodies used, see Table 2. Following incubation in secondary antibodies, slices were washed twice for $20 \mathrm{~min}$ in blocking solution, then 3 times for $20 \mathrm{~min}$ in $0.02 \mathrm{~mm}$ PBS. Washed slices were mounted on gelatin-coated glass slides with Vectashield mounting medium (Vector Laboratories) under glass coverslips sealed with nail polish.

Microscopy and image analysis. We sampled our data from dendrites of non-stellate-shaped neurons. Neurons were approximately uniformly sampled from all parts of the MSO, only sparing the most extremal portions close to the boundaries ( $~ 10 \%$ of the spatial extent). Staining of axons and other targets was initially assessed using an upright epifluorescence microscope (Eclipse 60i, Nikon, or Axio Imager.M1, Carl Zeiss) with $10 \times, 0.3 \mathrm{NA}$ and $20 \times, 0.5 \mathrm{NA}$ air objectives. Successfully stained sections containing at least one MSO nucleus were subsequently used for confocal imaging.

Images of stained tissue were acquired using a TCS SP5 confocal microscope system (Leica Microsystems). For line scan analysis of staining in the MSO, single images were acquired using a $20 \times, 0.7$ NA generalist immersion objective, adjusted for use with glycerol. Images were averaged 6-8 times by row of the image. Higher-magnification images were acquired with a $63 \times, 1.3 \mathrm{NA}$ glycerol immersion objective. Image stacks were acquired with a distance between sections approximately equal to the width of one pixel of a section. Typically, images of $1024 \times$ 1024 pixels were created, giving a pixel size of $240.50 \mathrm{~nm}$ with a distance of $251.77 \mathrm{~mm}$ between sections. All images in stacks were averaged 4-6 times by row. The number of images in a stack was chosen based on the depth of histologic staining and positions of dendrites and axons, and so was different for each stack. These stacks were used for following the path of axons in brain slices. Chromatic aberrations in image stacks were corrected using custom scripts in ImageJ. 

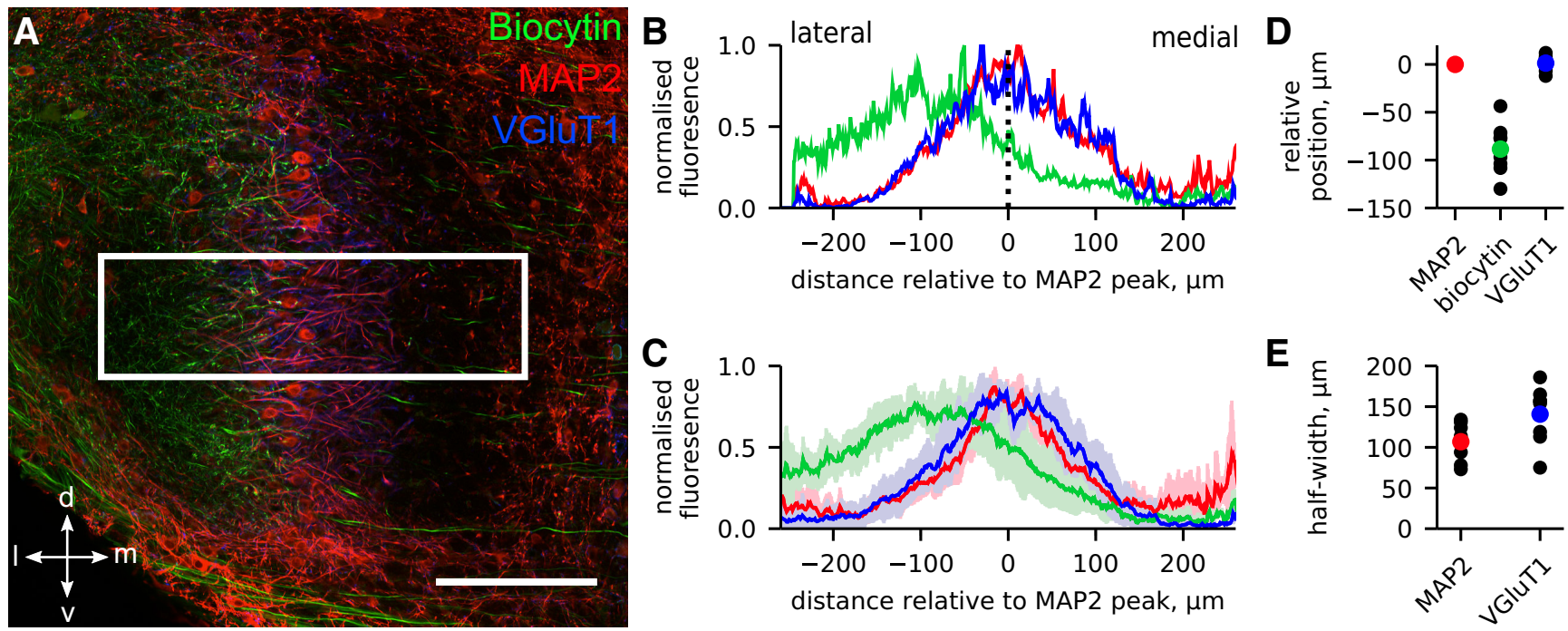

Figure 1. A, Single optical section showing the entire MSO, excitatory inputs from the cochlear nucleus, and staining for VGluT1. The boxed area was used for line scans (left to right, averaged across the vertical axis). Scale bar, $200 \mu \mathrm{m}$. $\boldsymbol{B}$, Normalized fluorescence values for MSO image shown in $\boldsymbol{A}$. Colors correspond to those in $\boldsymbol{A}$. C, Averaged values from 8 images (4 animals) of the MSO ipsilateral to the biocytin application. Each image is from a distinct physical slice. Multiple $(3,2,2,1)$ slices were used from single animals. $\boldsymbol{D}$, Peak fluorescence values relative to the MAP2 peak. Peaks are determined by fitting a Gaussian function to the fluorescence measurements. $\boldsymbol{E}$, Half-widths of Gaussian functions fit to measurements from each image.

Initial image processing to generate image stacks by acquisition channel was conducted using ImageJ. Line scan analysis was conducted using Igor Pro (WaveMetrics). Measurements of axons and dendrites were obtained using MetaMorph image analysis software (Molecular Devices). Measurements were initially recorded in Microsoft Excel and were subsequently analyzed with Python. Details of analysis are provided where relevant in Results.

Biocytin filling of excitatory inputs to MSO. In the experimental approach we used, many cells in the cochlear nucleus were unspecifically filled with biocytin (Seidl and Rubel, 2016) and stained with fluorophore-conjugated streptavidin. MSO neurons were visualized by staining for microtubule-associated protein 2 (MAP2). Additionally, tissue slices were costained for vesicular glutamate transporter 1 (VGluT1) to visualize excitatory synaptic terminals. Low-magnification images show axons projecting to the MSO (Fig. 1A). Upon approaching the dendritic field of MSO neurons, axons form many branches, which do not continue past the MSO cell somata.

Our qualitative observations were reinforced by line scans of fluorescence levels parallel to the path of the axons (Fig. 1B). The location of peak fluorescence was determined by fitting a Gaussian function to the data for each image. The peak of the MAP2 Gaussian fit was defined as zero. Biocytin-associated fluorescence peaks earlier than MAP2-associated fluorescence and decreases past the peak of MAP2 (Fig. 1C,D). This result indicates that axons terminate on the nearer dendrite (i.e., the lateral dendrite for the ipsilateral MSO), which was also apparent in higher-magnification images, and is expected from previous studies (Stotler, 1953). Some axons terminated past the somatic layer on the opposite dendrite (medial dendrite for the ipsilateral MSO), although these were the exception and were not considered further for detailed analysis. Additionally, some axons could be observed passing through the MSO and continuing toward the midline (Fig. 1A). The position of VGluT1 fluorescence was similar to that of MAP2, with both having the same peak position (Fig. 1D). The half-widths of Gaussian fits were, however, somewhat wider for VGluT1 than for MAP2 (141 $\mu \mathrm{m}$ vs $106 \mu \mathrm{m}$, one-tailed Wilcoxon signed rank test: $p=0.004$, signed rank $=36, N=8$; Fig. $1 E$ ), indicating a broad distribution of excitatory synapses to the distal dendrites.

Bouton identification was based on size and shape. Distinct varicosities in the axon, which were clearly larger than the neighboring axon and were adjacent to a MAP2-stained MSO neuron, were assumed to be synaptic boutons. Orthogonal optical slices through the image stack were inspected to ensure a bouton could be unambiguously identified as an extension of a particular axon. Only axons where the axon and all of its associated synaptic boutons could be clearly separated from surrounding axons were used for measurements.
To further verify that we were correctly identifying excitatory synapses, we looked more closely at individual boutons. As immunohistochemical staining does not reliably stain all the target protein present in a tissue sample, we produced average images from 60 synaptic boutons from 3 gerbils (see Fig. 2B). ROIs, centered on synaptic boutons, were selected from three channel images, and the average pixel intensity of these 60 images was calculated to produce an "average" bouton. These tissue samples had been counterstained for VGluT1 and MAP2 or GlyT2 and MAP2, and average images were also generated from these channels from the specified ROIs.

Bouton specificity was demonstrated by increased VGlut1 intensity only in bouton, but not axonal, regions by comparing the average fluorescence intensity ratio in a 5 pixel center circle to a 15 pixel annulus circle using the MetaMorph 7.1. (Molecular Devices) offline analysis tools.

Computational modeling. A minimal, multicompartmental model of an MSO neuron was simulated using MATLAB. The model consisted of a somatic compartment sandwiched between 10 dendritic compartments on each side. Each compartment was cylindrical with a length of $30 \mu \mathrm{m}$ for the somatic compartment and $15 \mu \mathrm{m}$ for each dendritic compartment to approximate the length of MSO dendrites (Rautenberg et al., 2009). The somatic compartment had diameter $15 \mu \mathrm{m}$ and the dendrites tapered linearly, with the most proximal compartment having diameter $4.4 \mu \mathrm{m}$, and the most distal $1.7 \mu \mathrm{m}$. The specific axial resistance connecting the compartments was taken as $200 \Omega \mathrm{cm}$, and the specific membrane capacitance as $\mathrm{C}_{\mathrm{m}}=0.9 \mu \mathrm{F} / \mathrm{cm}^{2}$ (Gentet et al., 2000). The resulting total cell capacitance was $\sim 40 \mathrm{pF}$.

The membrane potential, $\mathrm{V}$, of each compartment was modeled following a Hodgkin-Huxley type equation as follows:

$$
C_{m} \frac{d V}{d t}=-\left(I_{\mathrm{KLT}}+I_{\mathrm{HCN}}+I_{\text {leak }}+I_{\text {axial }}+I_{\text {syn }}\right)
$$

where $\mathrm{I}_{\mathrm{KLT}}$ is a low threshold potassium current modeled according to Mathews et al. (2010), $\mathrm{I}_{\mathrm{HCN}}$ is an HCN channel current modeled according to Baumann et al. (2013), and $\mathrm{I}_{\text {leak }}=0.05 \mathrm{mS} / \mathrm{cm}^{2}$. The axial current, $\mathrm{I}_{\text {axial, }}$ was determined as follows:

$$
I_{\text {axial }}^{i}=\frac{V_{i}-V_{i-1}}{R_{\text {axial }}^{i-1, i}}+\frac{V_{i}-V_{i+1}}{R_{\text {axial }}^{i+1, i}}
$$

EPSC $I_{\text {syn }}$ was modeled according to Lehnert et al. (2014) as an Ohmic current with the time course of synaptic conductance modeled as the following double exponential function: 


$$
G_{\text {exc }}(t)=g_{\text {exc. } \max } \frac{\left(1-e^{-t / 1.0}\right)^{1.3} e^{-t / 0.27}}{\max \left(\left(1-e^{-t / 1.0}\right)^{1.3} e^{-t / 0.27}\right)}
$$

The peak conductance of the low threshold potassium current (in $\mathrm{mS} / \mathrm{cm}^{2}$ ) was dependent on distance from the soma center according to the following:

$$
G_{K L T}(x)=57\left(1+1.5^{-x / 22}\right)
$$

with $x$ in micrometers. For the $I_{\mathrm{HCN}}$ current, the peak conductance was uniform throughout the cell at $1.26 \mathrm{mS} / \mathrm{cm}^{2}$. The conductance amplitudes were chosen to fit the physiological resting potential $(-68 \mathrm{mV})$ and input resistance (peak: $9 \mathrm{M} \Omega$; steady state: $6 \mathrm{M} \Omega$ ) as measured at the soma in MSO neurons (Couchman et al., 2010; Nabel et al., 2019). The reversal potential of potassium was taken as $-90 \mathrm{mV}$, of the $I_{\mathrm{HCN}}$ as $-35 \mathrm{mV}$, of the leak current as $-70 \mathrm{mV}$, and of excitatory synaptic input as $0 \mathrm{mV}$. Although there is evidence for high threshold potassium currents in MSO neurons, which can influence membrane response properties (Nabel et al., 2019), the essential features of MSO membrane potential behavior can be replicated with only low threshold potassium currents (Mathews et al., 2010; Winters et al., 2017; Nabel et al., 2019).

For the subset of simulations with multiple input fibers per dendrite, we included stochastic synaptic transmission. We assume a readily releasable pool of $\mathrm{R} \leq \mathrm{R}_{0}$ vesicles for every bouton that is depleted on a presynaptic spike with release probability $p_{R}$ : After every input spike a, we draw a random variable $0 \leq \mathrm{k} \leq \mathrm{R}$ from the binomial distribution $\mathrm{B}\left(\mathrm{k}, \mathrm{p}_{\mathrm{R}}, \mathrm{R}\right)$ and set the new pool size to $R-k$. Afterward, the pool is replenished exponentially with interstimulus interval, i.e., $\mathrm{R} \leftarrow \mathrm{R}_{0}+\left((\mathrm{R}-\mathrm{k})-\mathrm{R}_{0}\right)$ $\exp \left(-\mathrm{ISI} / \tau_{\mathrm{R}}\right)$, before the next binomial random value $k$ is drawn. The integer number $k$ of released vesicles is then multiplied by the fiber conductance divided by the maximum pool size per fiber $\mathrm{R}_{0} \mathrm{~N}_{\mathrm{b}}$ and used as an input to the neuron model at the specific dendritic segment. Following Couchman et al. (2010), we set the parameters $\mathrm{p}_{\mathrm{R}}=0.45, \tau_{\mathrm{R}}=30 \mathrm{~ms}$, and $\mathrm{R}_{0}=118 / \mathrm{N}_{\mathrm{b}}$, where $N_{b}$ is the number of boutons per fiber. All simulations with stochastic synapses have been performed with $N_{b}=4$, with the four axo-dendritic contact sites randomly and uniformly distributed on the 10 dendritic segments. In all simulations with inputs from multiple fibers, we sampled fiber-specific temporal offsets equally spaced from an interval of duration $J$. The parameter $J$ is further called imprecision; that is, all spikes from one fiber are delayed by (or lead) the spikes of a given other fiber by the same amount of time.

TEM. A male gerbil (P37) was perfused with $2.5 \%$ glutaraldehyde in $0.1 \mathrm{M}$ cacodylate buffer, $\mathrm{pH} 7.2$; the brain was dissected and postfixed for $12 \mathrm{~h}$ in the same fixative at room temperature and cut into horizontal vibratome sections $(100 \mu \mathrm{m})$. Sections containing the $\mathrm{MSO}$ were postfixed in $1 \% \mathrm{OsO}_{4}$ solution
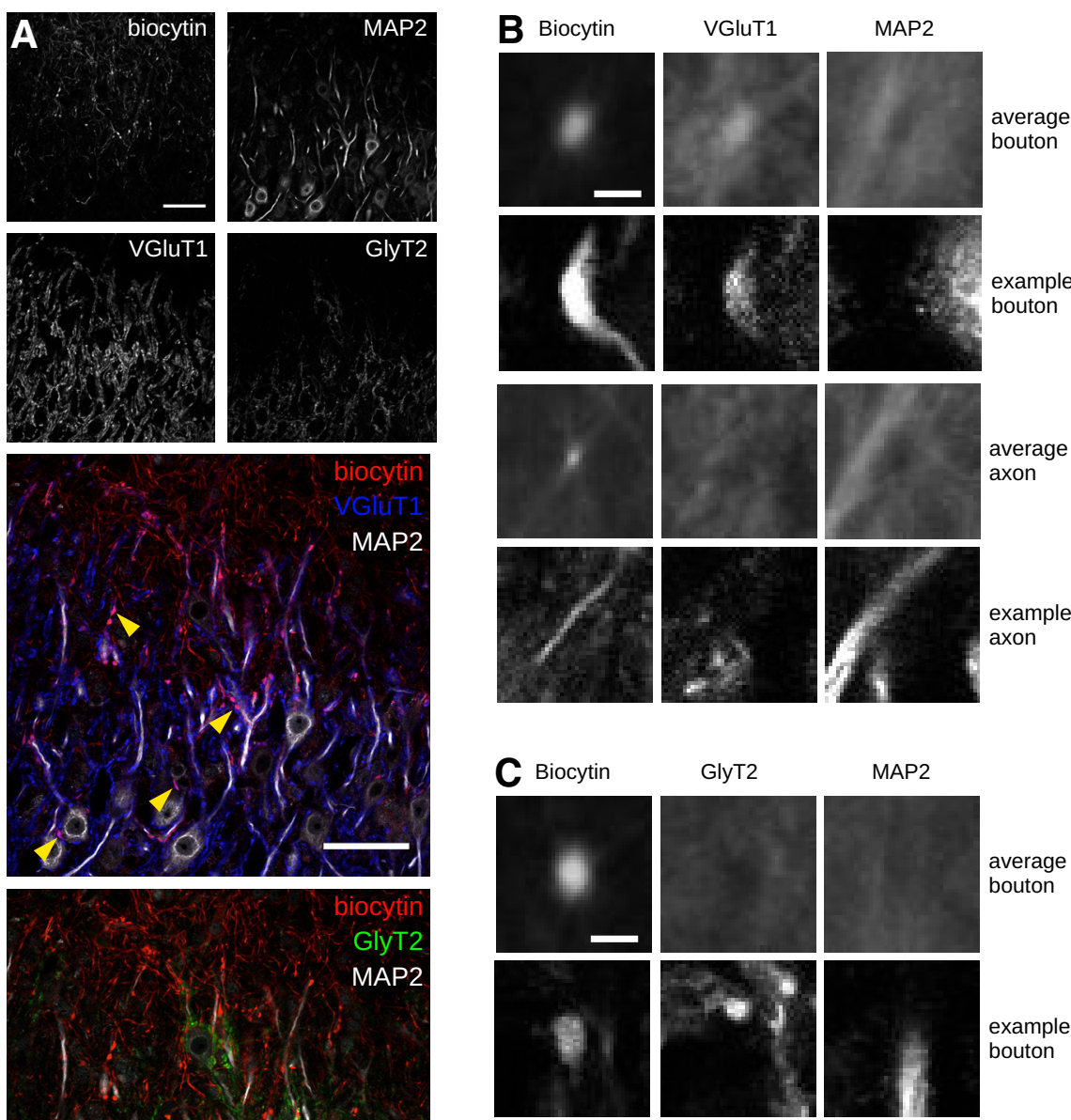

GlyT2

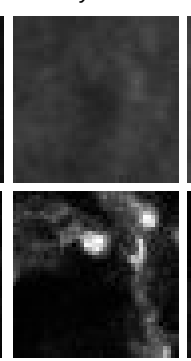

MAP2
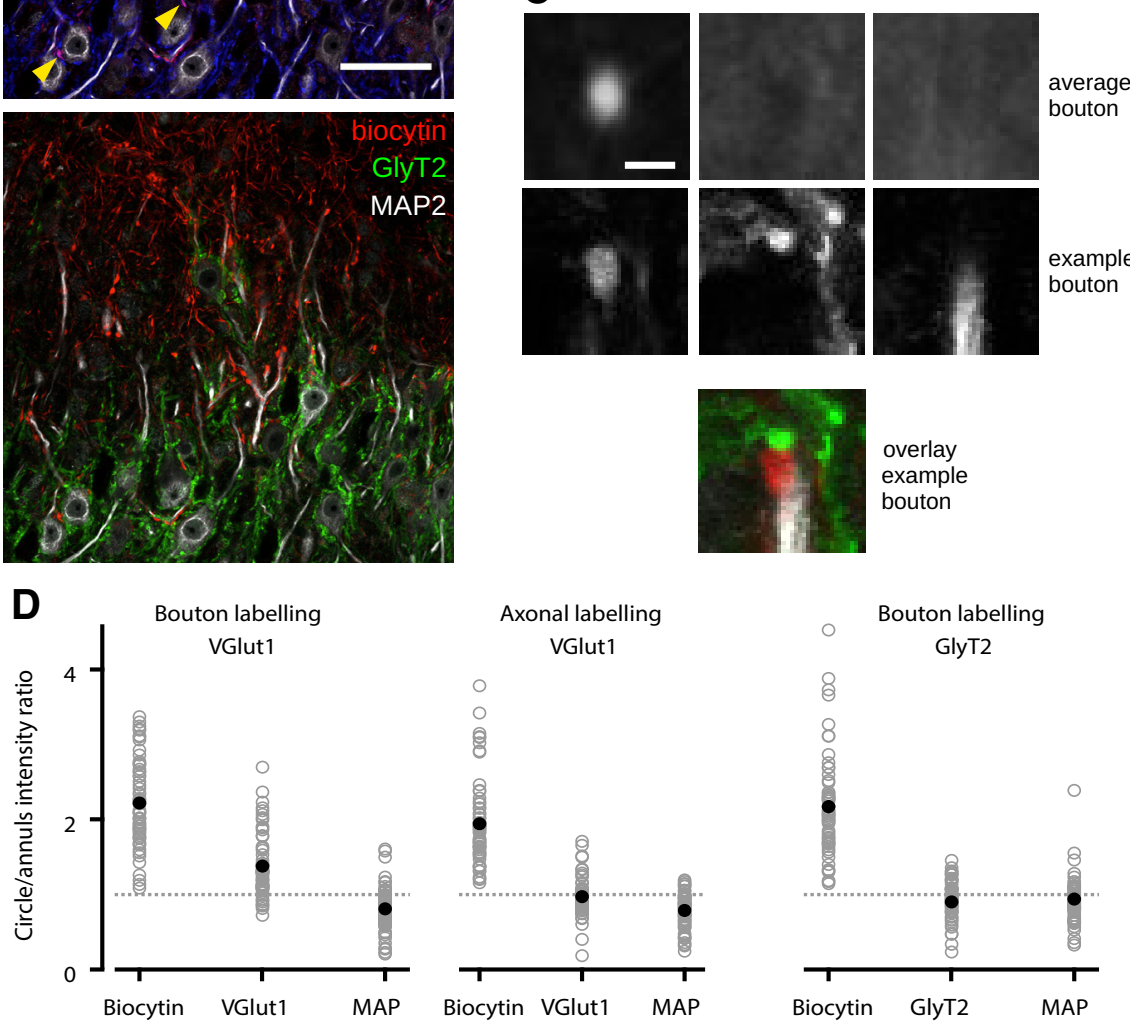

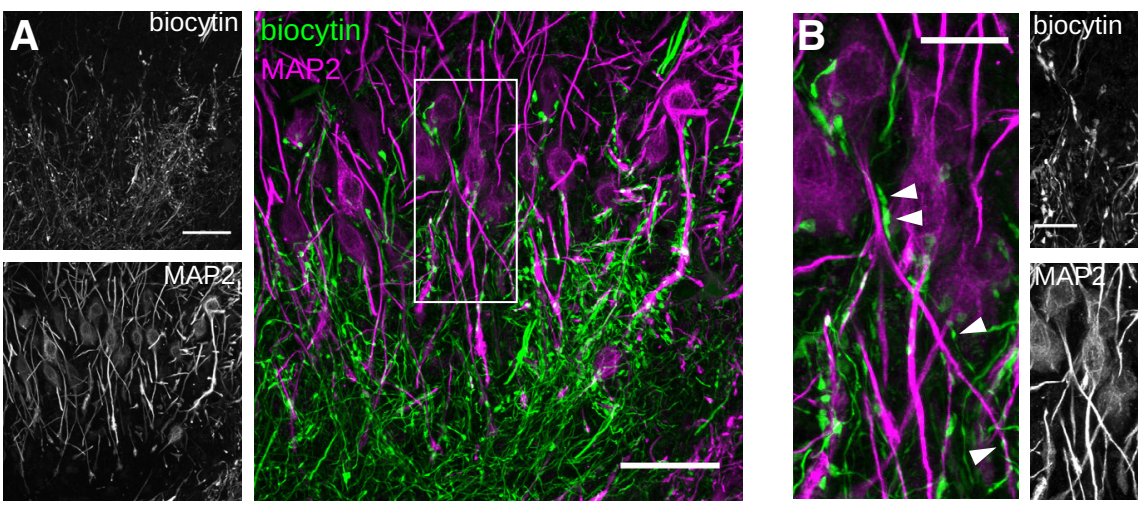

C Number of boutons per axon
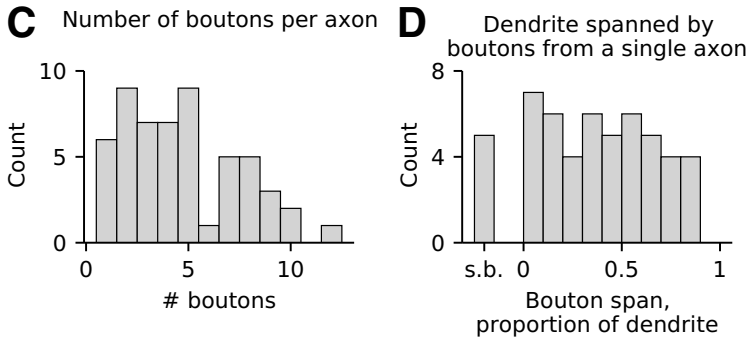

\section{$E$}

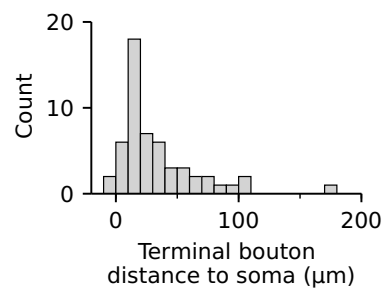

Materials and Methods). Many putative synaptic boutons were visible on these terminating axons in close apposition to MSO neurons. Biocytin-filled boutons commonly overlapped with VGluT1 staining (examples indicated in Fig. $2 A)$. Overlap of boutons with GlyT2 staining was not observed, consistent with glycinergic inputs originating in the medial nucleus of the trapezoid body but not in the cochlear nucleus. Additionally, we never observed a pattern of somatic innervation like that seen in previous studies of glycinergic innervation of MSO neurons by the medial nucleus of the trapezoid body (Werthat et al., 2008).

Many, but not all, of the synaptic boutons showed overlapping VGluT1 staining (Fig. 2B,D). The average VGluT1 stain was very similar to the average bouton (see Materials and Methods), except for having more staining throughout the image because VGluT1 stains many more synaptic boutons than are filled with biocytin. The average MAP2 staining showed a darker patch in the center of the image because of boutons commonly appearing adjacent to dendrites and somata. Repeating the selection and averaging process with stretches of axon that did not include boutons showed a random pattern in the VGluT1 staining, with no corre-

for $1 \mathrm{~h}$ on ice, rinsed in buffer, dehydrated in a graded acetone series, and embedded in epoxy resin (Epon). The ROI was found on superficial semithin sections ( $1 \mu \mathrm{m}$, RMC MT-7000 microtome) stained with Richardson's reagent; then a short ultrathin section series (50 slices at $50 \mathrm{~nm}$ ) was cut with a Leica Microsystems EM UC6 ultramicrotome, mounted on formvar-coated copper grids, and subsequently contrasted with $8 \%$ lead citrate and $4 \%$ uranyl acetate. The grids were examined with a FEI Morgagni $268 \mathrm{TEM}$ at $80 \mathrm{kV}$ : the ROI was photographed at $11 \mathrm{kx}$ for overview and stitched with the Adobe PS Photomerge tool. A series of 25 neighboring planes was aligned for $3 \mathrm{D}$ reconstruction, and surface renderings were generated after manual segmentation with Amira 5.7 software. Additionally, synaptic sites were photographed at $22 \mathrm{kx}$ on several neighboring slices. Color outlines and shading were added manually using Adobe PS.

Experimental design and statistical analysis. Animal ages and sexes are specified in the above sections for each experiment. The size of the dataset $(N=55)$ was chosen to obtain a good estimate for the mean bouton count per axon in the sense that the $95 \%$ CI should be $<2$.

Analysis of light microscopy images were performed on a set of $N=55$ axons. Axon numbers vary between figure panels, since not all analyses could be performed on all $N=55$ axons. The exclusion criteria were as follows: Figure $3 D, N=52,3$ axons were excluded in which we could not obtain a good length estimate of the dendrite; Figure $3 E$, $N=54,1$ axon was excluded where the cell soma was partly cut off; Figure $5 B, C, E, F, N=49,6$ axons with $<2$ boutons needed to be excluded; Figure $5 D, N=41,14$ axons needed to be excluded because they only had one bouton or were branching; Figure 6, $N=17$, axon branches could be considered.

The experiment has not been preregistered.

\section{Results}

Identification of presynaptic boutons on MSO dendrites Excitatory input fibers to MSO dendrites were visualized by biocytin application to the presynaptic cochlear nucleus (see spondence to the averaged filled axon (Fig. $2 B, D$ ). Repeating this process with tissue costained for GlyT2 showed no overlap between GlyT2 staining and boutons (Fig. 2C,D). Instead, the average GlyT2 image shows a dark patch corresponding to the location of the average bouton. Overall, this analysis shows that we correctly identified excitatory synaptic boutons terminating on MSO neurons.

\section{Pattern of excitatory inputs to MSO neurons}

We sought to quantify the pattern of synaptic boutons formed by individual axonal branches. We identified 55 terminal axon branches in tissue taken from 6 gerbils, each of which formed one or more synaptic boutons in apposition to MSO dendrites (Fig. $3 A, B$ ). Each of these axons approached the dendrite, then ran adjacent to it for some distance in the dendro-somatic direction before terminating with a terminal bouton closer to the soma. Only those axons where we could identify the point at which the axon first approached the dendrite were included in our measurements. Most axons (49 of 55) formed boutons along the dendrite in addition to the terminal bouton, with a median of four boutons for all 55 axons (Fig. 3C). We measured the length of the MSO dendrite spanned by boutons from a single axon, that is, the distance along the dendrite between the terminal bouton and the most distally located bouton. This distance varied from the size of the bouton for single bouton axons, up to $120 \mu \mathrm{m}$. The proportion of the dendrite spanned by boutons from a single multibouton axon varied unsystematically, with no clear peak in the distribution of values (Fig. 3D). Although boutons from single axons could span large portions of the dendrite, axons tended to terminate with a terminal bouton at the soma or proximal dendrite. The majority (39 of 54, one unknown where soma was outside of the image) of terminal boutons occurred 

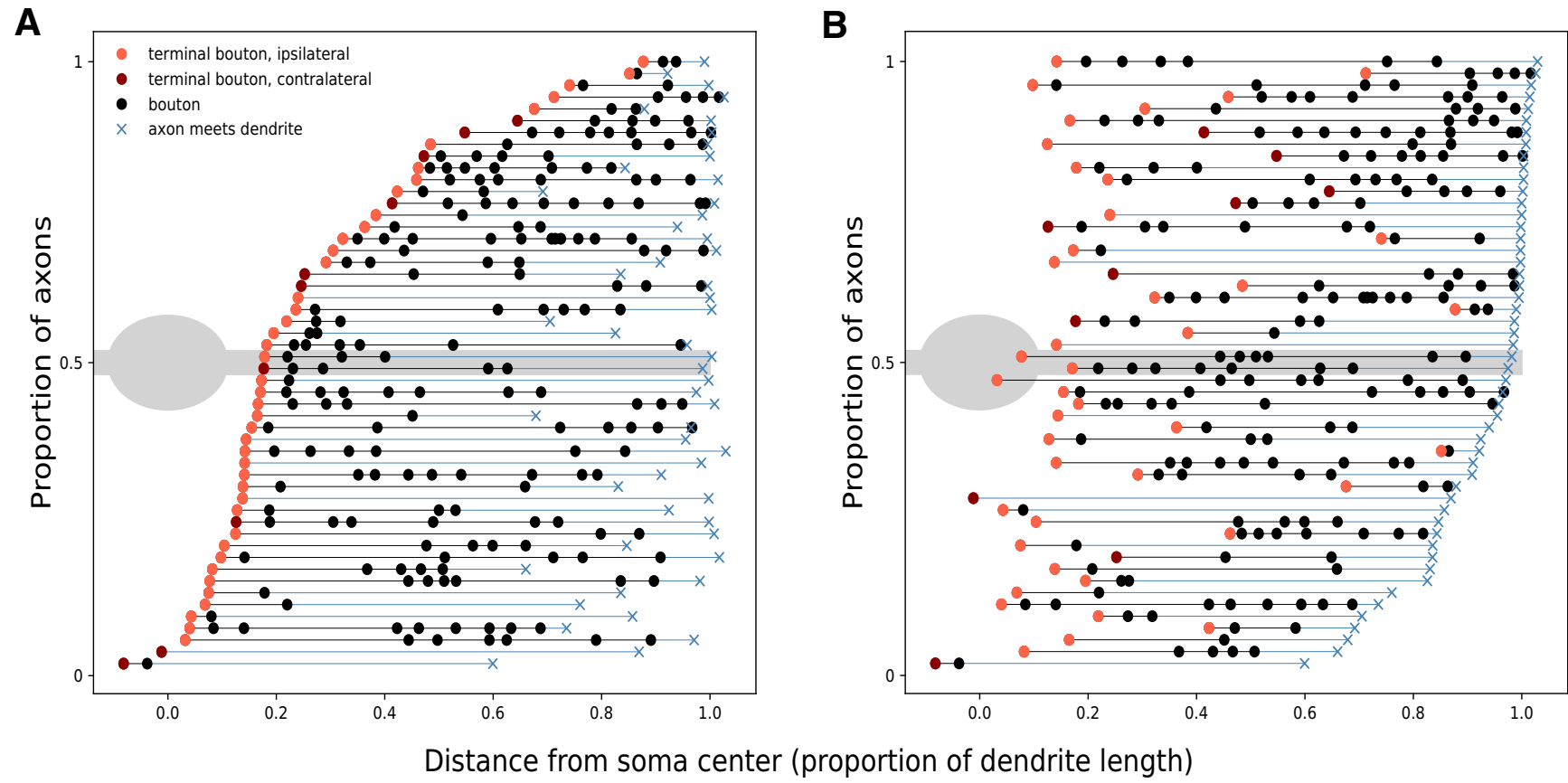

Figure 4. Overview of axon measurements. $\boldsymbol{A}$, Each dendrite-axon pair is ordered according to the position of the terminal bouton. Dendritic length (normalized) is measured from the soma center. For branching axons (see Fig. 6), all boutons are shown on a single line. "Axon meets dendrite" position is the sum of the distance at the most distal bouton and the length of the bouton-free part. Distance values $>1$ thus reflect measurement errors. $\boldsymbol{B}$, Same as in $\boldsymbol{A}$ ordered according to "axon meets dendrite" point. As a criterion for the meeting point, we used a distance threshold of $5 \mu \mathrm{m}$.

within $40 \mu \mathrm{m}$ of the soma center (Fig. $3 E$ ). An overview of these data, showing normalized positions of all boutons, is given in Figure 4.

The length of dendrite spanned by boutons from a single axon was greater for axons with more boutons (Fig. 5A, $B$; linear regression, $R^{2}=0.46, p=9.2 \times 10^{-8}, N=49$ axons). Boutons were not, however, evenly spaced throughout this length. Clusters of boutons were common (e.g., Fig. 5A), as were long stretches of axon between clusters. The distribution of interbouton distances was skewed toward smaller values (Fig. 5C). These data were not well modeled as a Poisson process (Lilliefors' test, comparison to exponential distribution, $p<0.001$, Kolmogorov-Smirnov $=0.20$, $N=192$ intervals from 49 axons). This suggests that the distances between boutons are not random but instead depend on the locations of other boutons on the axon.

Axons innervating MSO neurons typically "followed" the dendrite. The terminal axonal branches we observed were positioned parallel to the dendrites they innervated and rarely made excursions away from the dendrite (example in Fig. 5A). We compared the distance on the dendrite spanned by the boutons from one axon to the distance on the axon itself between the initial and terminal boutons. The axonal distances were slightly but consistently larger than the dendritic distances (medians 51.2 vs $50.5 \mu \mathrm{m}$, one-tailed Wilcoxon signed rank test, $p=1.8 \times 10^{-4}$, signed $\mathrm{rank}=88, N=41$ axons). Least squares linear regression indicated the dendritic span was a good predictor of the axonal span (slope $=1.02, r^{2}=1.00, p=1.5 \times 10^{-53}, N=41$ axons; Fig. $5 D$ ). Upon meeting the dendrite, axons typically ran adjacent to the dendrite for some distance before the first bouton. We termed this section of axon without boutons the "tail." Tail lengths varied from $0 \mu \mathrm{m}$, where the axon formed a bouton immediately on contacting the dendrite, to $95.7 \mu \mathrm{m}$, with a median of $18 \mu \mathrm{m}$ (Fig. $5 E$ ). The distribution of these values shows that many axons converge on dendrites at relatively distal dendritic positions, and then run parallel for some distance before forming the first bouton (see also Fig. 4). A least squares linear regression indicates a weak but statistically significant correlation between tail length and bouton span (Fig. 5F; slope $=-0.37, r^{2}=0.20$, $p=0.001, N=49$ axons), which is to be expected, since longer tails would leave less space remaining along the length of the dendrite for the bouton-forming part of the axon. The large fraction of small tail lengths for all bouton spans (Fig. $5 F$ ) lets us rule out that long tails predominantly reflect missed distal boutons.

Terminal axonal branches exhibit minimal branching. Of the axons we identified, 13 of 55 possessed additional branches, all of which exclusively targeted the same dendrite as the main branch of the axon (example in Fig. 6A). In most cases (10 of 13), only one additional branch was observed (Fig. 6B). Branches also tended to be relatively short, with a median length of $9 \mu \mathrm{m}$, or $12 \%$ of the total length of axon adjacent to the dendrite. Branch lengths did not correlate with the overall length that the axon was adjacent to the dendrite (Fig. 6C). We did observe axonal branches targeting other dendrites, as expected from previous studies (Smith et al., 1993; Beckius et al., 1999), but the branch points in these cases occurred before the axon innervating any dendrite with synaptic boutons.

We also sought to determine the overall distribution of boutons throughout the MSO dendrite. For this analysis, we identified MSO dendrites and measured the position of each bouton on that dendrite, regardless of whether we could follow the associated axons (e.g., in Fig. $7 A$ ). We measured the positions of 241 boutons on 18 lateral dendrites and 164 boutons on nine medial dendrites. The number of boutons per dendrite was between 5 and 29 (median $=15)$. Despite that not all identified varicosities may be functional synapses, these numbers are likely an underestimate of the true number of boutons for each dendrite, given the unlikelihood that all cochlear nucleus neurons were filled with biocytin. Nevertheless, combining measurements from many dendrites should represent the distribution of all boutons on a single dendrite, assuming the studied MSO population to be 

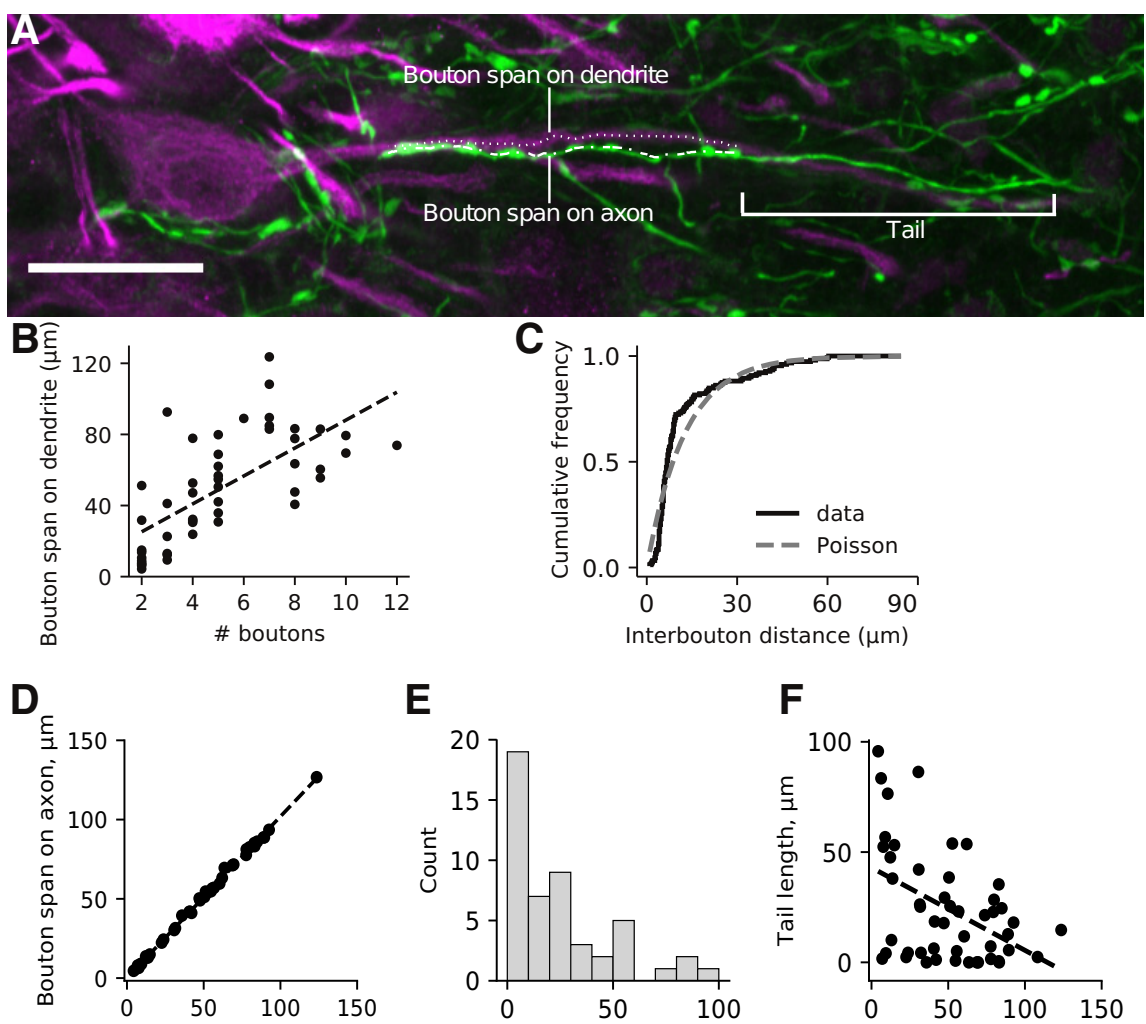

Bouton span on dendrite, $\mu \mathrm{m}$
E

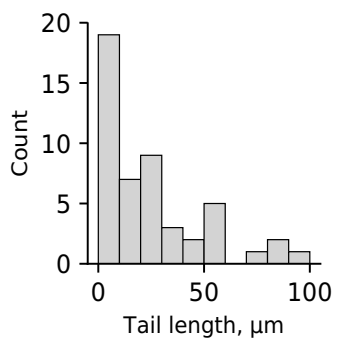

$\mathbf{F}$

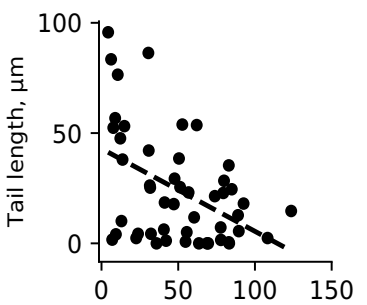

Bouton span on dendrite, $\mu \mathrm{m}$
Figure 5. A, Axons tend to follow dendrites closely, and synaptic boutons often occur in clusters. The spans of boutons as measured on the dendrite and on the axon are indicated. The length of axon between the first axo-dendritic contact point and the first bouton is referred to as the "tail." Scale bar, $30 \mu \mathrm{m}$. $\boldsymbol{B}$, The span of boutons along the dendrite can be explained to some extent by number of boutons. Least squares linear regression, $R^{2}=0.46, p=9.2 \times 10^{-8}, N=49$ axons. $C$, Cumulative frequency of distances between boutons could not be modeled as a Poisson process, suggesting that boutons are not randomly spaced relative to other boutons. $\boldsymbol{D}$, The span of boutons as measured on the afferent axon was almost identical to the span on the dendrite. Least squares linear regression slope $=1.0, r^{2}=1.02, p=1.5 \times 10^{-53}, N=41$ axons (branching axons excluded). $\boldsymbol{E}$, Distribution of tail lengths $(N=49$ axons). $\boldsymbol{F}$, Tail length versus bouton span shows significant negative correlation ( $N=49$ axons). All data were collected from 6 animals.

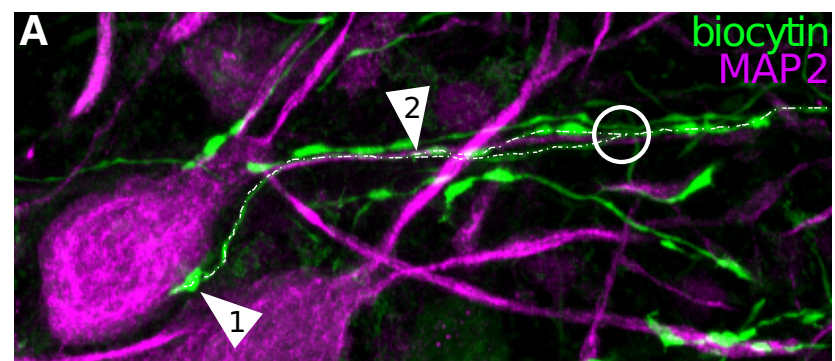

B

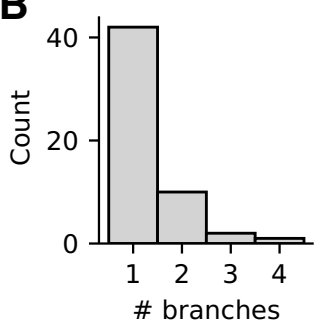

$\mathrm{C}_{\overline{\mathrm{S}}}$

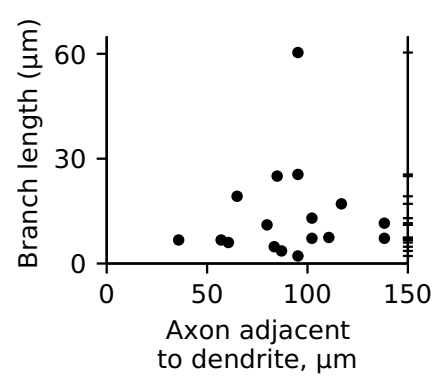

Figure 6. Axons branch in parallel to dendrite. $\boldsymbol{A}$, Example of a branching axon, which has been traced over with a dashed line. Terminal boutons of each branch are indicated with arrows 1 and 2, and the branch point is circled. $\boldsymbol{B}$, Axon branching was visible in $20 \%$ of measured axons. \# branches is total number of branches, so one branch means zero branch points. $\boldsymbol{C}$, Distribution of branch lengths is visualized on the right axis. Branch lengths did not correlate to the total length of axon adjacent to the dendrite. Pearson's $r=0.075, p=0.78, N=17$ axon branches. homogeneous in this respect. Bouton position was measured as distance from soma center, and normalized to the length of the dendrite. Two boutons were included that were positioned on the opposite side the soma center (and so have a negative position) because they were clearly associated with axons innervating the dendrite under inspection. For each neuron, only the boutons along the longest visible dendritic branch were included in the analysis, ignoring boutons on other branches. For these main single dendritic branches, the distribution of boutons exhibits an approximately uniform distribution (Fig. 7B). Additionally, the distribution did not differ between ipsilateral and contralateral MSO dendrites (Kolmogorov-Smirnov test, $p=0.83$, Kolmogorov-Smirnov $=0.063, N=$ 241 and $N=164$ boutons, respectively). Given that all MSO neurons exhibit some degree of dendritic branching (Rautenberg et al., 2009), we expect the overall number of boutons on the dendrite is greater distal to dendritic branch points.

\section{Functional significance of distributed excitatory inputs}

We next assessed the effects of the observed spatial arrangement of presynaptic contact sites on the function of MSO neurons, specifically the dendritic propagation delay, with a computational model. To test whether the dendritic propagation delay might affect ITD tuning, we simulated a multicompartmental model of an MSO neuron with simplified dendritic geometry (see Materials and Methods; Fig. 8A), which reproduced propagation delays measured experimentally (Fig. 8B). Propagation delay was measured in the model as the relative timing of $50 \%$ maximum amplitude of the propagating EPSP between the stimulated compartment and the somatic compartment. We then simulated trains of bilateral excitatory postsynaptic conductances with different positions on the dendrites, at a range of amplitudes and frequencies (as indicated in Fig. $8 D$ ). Synapses were modeled to contact at the distal dendrites, at the proximal dendrites, or offset, with one distal and one proximal input (Fig. 8A). The response to dual distal and dual proximal inputs varied only in amplitude, but when the inputs were spatially offset, the peak of the response function was shifted by up to $150 \mu$ s (Fig. 8C,D). The magnitude and direction of the shift varied depending on the strength and the frequency of synaptic input (Fig. 8D).

These results suggest that physiological dendritic conduction delays induced by the spatial location of presynaptic boutons could indeed affect the ITD response function of MSO neurons; thus, we used our model MSO neuron to investigate how the excitatory input pattern might influence the physiological function of these neurons.

We first looked at whether distributing synaptic input over a larger extent of the dendrite would affect the somatic response. We simulated two configurations: one where the synaptic input was localized at just the one compartment closest to the soma, 

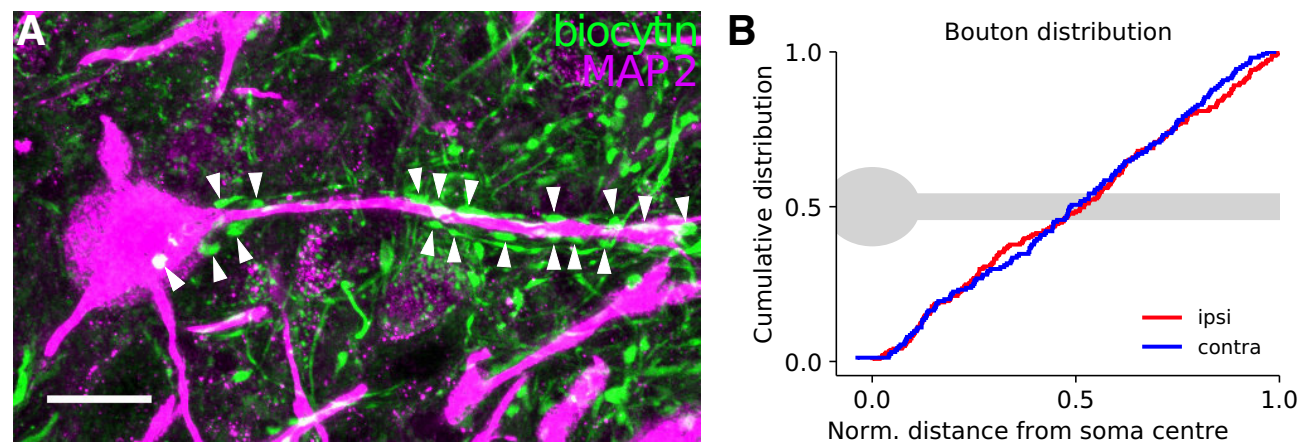

Figure 7. Symmetry of bouton distributions. $\boldsymbol{A}$, For some MSO neurons, the positions of all boutons were measured, indicated with white arrows in example given. $\boldsymbol{B}$, Cumulative plot shows similar, uniform distributions of boutons along the MSO dendrite (indicated in gray) for both ipsilateral and contralateral inputs.

and one where it was distributed across the whole dendrite (Fig. 9A). To be able to compare effects of anatomy, the total synaptic conductance was identical between localized and distributed versions of the model at $37 \mathrm{nS}$ (Couchman et al., 2010). To assess the effect of a conductance increase for multiple boutons, we also simulated EPSPs for a single proximal bouton with average physiological conductances 37/4 $\mathrm{nS}$, assuming the median of four boutons per fiber (weak). For the distributed synaptic input, the conductance $37 \mathrm{nS}$ was divided equally between the compartments receiving synaptic stimulation. For all input configurations, the model was simulated twice with different simulated axonal conduction velocities. A "fast" version with conduction velocity $=10 \mathrm{~m} / \mathrm{s}$ was used to simulate the presence of myelin (Smith et al., 1993; Sinclair et al., 2017), while a "slow" version with conduction velocity $=1$ $\mathrm{m} / \mathrm{s}$ was used to simulate an unmyelinated axon (Weidner et al., 1999; Meeks and Mennerick, 2007). The onset timing of the synaptic conductance was the same for all versions of the model.

Distributing the synaptic conductance along the dendrite resulted in a distinctly different EPSP waveform at the soma (Fig.

9A). Distributed synapses evoked larger EPSPs than localized synapses (even with equal fiber conductance) in every dendritic compartment for both fast and slow axonal conduction velocities (Fig. 9B,C). Distributed synapses differed from localized synapses according to three different EPSP properties: amplitude, halfwidth, and latency. Distributed synapses tended to produce somatic EPSPs that were slightly larger (up to $1.7 \mathrm{mV}$ ) and briefer (up to $170 \mu$ s smaller half-width) compared with synaptic input localized to single compartments (Fig. 9D1,D2). Additionally, the peak of the somatic EPSP occurred up to $110 \mu$ s earlier for distributed synapses than for localized synapses (Fig. 9D3), a physiologically relevant time difference for an MSO neuron, since the entire range of relevant ITDs in the gerbils is only $\sim \pm 120 \mu \mathrm{s}$. This difference was more pronounced for the slow axonal velocity condition. For the fast axonal velocity condition, there was a maximum peak advance of only $65 \mu$ s relative to the single input, which still is a functionally relevant time difference for an MSO neuron. This result suggests that myelin in terminal branches could play a small role in tuning peak timing in the MSO. EPSPs derived from physiologically "weak" single boutons are particularly attenuated at proximal locations (by a factor of $\sim 3$; Fig. $9 D 1$ ), and are generally broader and exhibit larger peak latency (Fig. 9D2,D3).

The larger membrane depolarization induced by distributed inputs generated larger low threshold potassium current $\left(\mathrm{I}_{\mathrm{KLTT}}\right)$. As $\mathrm{I}_{\mathrm{KLT}}$ is known to sharpen EPSPs in the MSO (Mathews et al., 2010), it can explain why distributed inputs can have both higher amplitudes and shorter half-widths, as well as earlier peak timing. We repeated our simulations using a static KLT model to test whether $I_{\text {KLT }}$ plays a role in generating the differences we observed. In this model, the gating variables of the KLT conductance are frozen at their steady-state resting values, eliminating dynamic changes in KLT conductance (Mathews et al., 2010). In this version of the model, somatic EPSPs were larger for all simulated synaptic inputs, and distributed inputs again elicited larger 
A

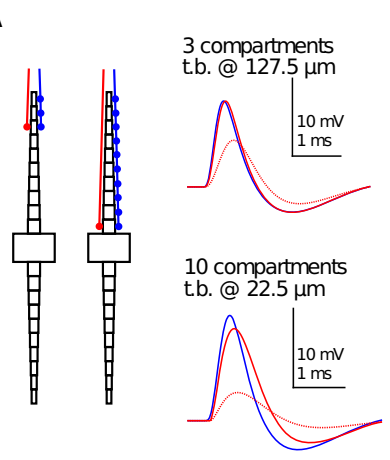

D1

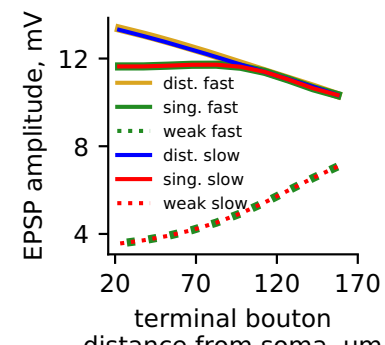

E1

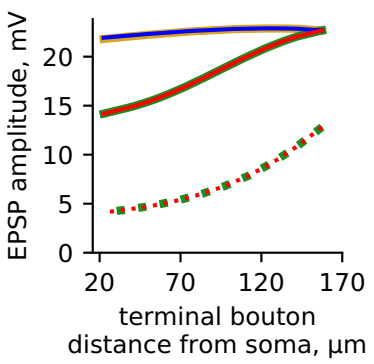

B

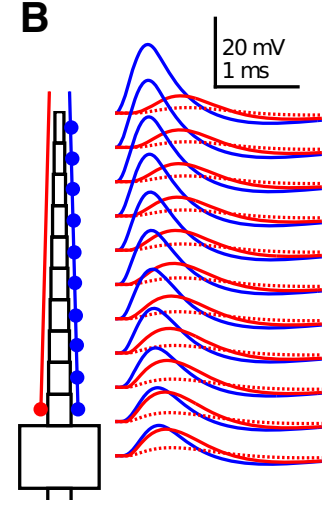

D2

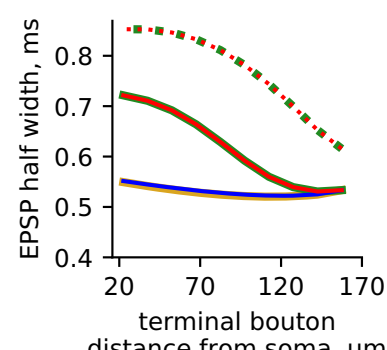

E2

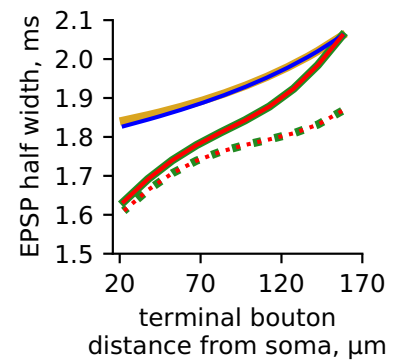

C

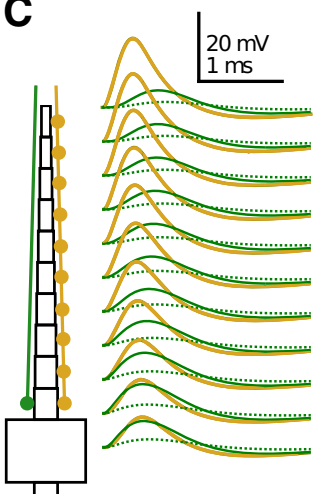

D3

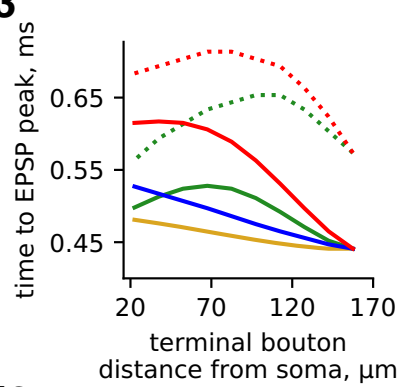

E3

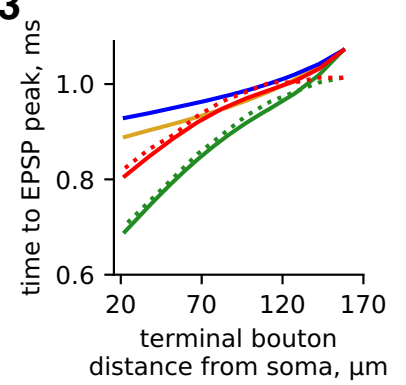

Figure 9. Distributed synapses facilitate EPSP sharpening. $A$, Two example configurations of synaptic input, shown on schematic of model geometry, with total conductance-matched (37 nS per fiber) single (red) and distributed (blue) synapses, and corresponding somatic membrane potential traces at right. Axonal conduction velocity $=1 \mathrm{~m} / \mathrm{s}$. t.b., Terminal bouton. Dotted traces stem from simulations with physiological average bouton conductance $37 / 4 \mathrm{nS}$ (weak) for comparison. $\boldsymbol{B}$, Membrane potential for each compartment on the synaptically active dendrite plus soma. Traces correspond to the adjacent compartment in schematic. Axonal conduction velocity $=1 \mathrm{~m} / \mathrm{s}$. C, Same as in $\boldsymbol{B}$, but with axonal conduction velocity $=10 \mathrm{~m} / \mathrm{s}$. $\boldsymbol{D}$, Somatic EPSP properties relative to position of single or terminal synaptic bouton. $E$, Somatic EPSP properties for a version of the model with $I_{K L T}$ frozen at resting conductance levels.

somatic EPSPs (Fig. 9E1). Contrary to the normal, dynamic KLT model, however, the EPSP half-widths were larger for distributed synapses, as expected given the reduced amount of $\mathrm{I}_{\mathrm{KLT}}$ available to sharpen the large EPSPs (Fig. 9E2). Static $\mathrm{I}_{\mathrm{KLT}}$ also reversed the trend in peak timing, with EPSPs from distributed synapses peaking later than EPSPs from single synapses (Fig. 9E3). Physiologically weak single synapses show smaller differences in EPSP shapes than for active KLT (Fig. 9E2,E3), however, the amplitude attenuation of a factor of $\sim 3$ for proximal bouton locations remains (Fig. 9E1). Thus, particularly for large-fiber conductances, the active channel properties of KLT transform the passive low-pass filtering dendrite into an anatomic compartment facilitating precise temporal integration.

The sharpening of the EPSP because of multiple synaptic contacts of a single fiber specifically exploits the highly precise temporal association of the synaptic release processes at all boutons. We therefore asked to what extent the sharpening depends on this temporal precision and, conversely, whether simultaneous release from boutons of multiple axons would achieve the same sharpening. By simulating EPSPs arising from inputs of four fibers with varying temporal offsets (imprecision as defined in
Materials and Methods), we found that the sharpening effect is considerably reduced for a temporal imprecision of $\geq 300 \mu \mathrm{s}$ (Fig. 10). Even single low-frequency anteroventral cochlear nucleus spherical bushy cells, despite their exquisite phase locking to acoustic stimulation, may still exhibit jitters of up to few hundreds of microseconds (Joris et al., 1994). Summing inputs from multiple spherical bushy cells would thus at least imply imprecisions in a similar range, probably even larger if the mean spike phases of bushy cells are not synchronized. Thus, the anatomic association of release sites at a single fiber may indeed help to confine temporal broadening of EPSPs in vivo. Nevertheless, even for large imprecisions, the EPSP-half-width is still lower than for a single synaptic site close to the soma (Fig. 10B), indicating that the spatially distributed activation of synapses alone already induces sharpening independent of whether those synapses arise from the same axon.

To see whether the effects we observed in our simulations of single-dendrite stimulation might translate to an effect on ITD coding, we simulated the model with inputs to both dendrites. A $400 \mathrm{~Hz}$ train of EPSGs was simulated, and the difference in input timing between dendrites was varied. We tested four different 
synaptic input configurations, each with a terminal bouton at the most proximal dendritic compartment. We compared a single site of synaptic input to distributed inputs over two, four, or six compartments, as well as to a single site with physiologically weak bouton conductance (Fig. 11A). The four compartment input configuration was designed to resemble our data and therefore simulated the median number of boutons, distributed over about half of the dendritic length, with a terminal bouton close to the soma. The response of the simulated somatic membrane potential for this configuration shows both higher maximum and lower minimum values compared with the single synapse (Fig. 11B). This indicates that larger EPSPs recruit more $\mathrm{I}_{\mathrm{KLT}}$ in the distributed synaptic configuration, leading to a lower effective membrane time constant. Varying the input timing between dendrites produces variations in peak somatic membrane potential that can be used as a proxy for the sensitivity of an ITD tuning function recorded from MSO neurons in vivo (Fig. 11C) (Brand et al., 2002). The peak membrane potentials were larger for more distributed inputs. As the overall range of values (maximum - minimum) was also larger, the slope of the voltage tuning curve was steeper (Fig. 11D). A single physiologically weak synaptic bouton per dendrite results in hardly functional ITD tuning.

To determine whether the variations in binaural integration between single and distributed synaptic input might be physiologically relevant, we calculated a Fisher-information-derived sensitivity measure of the $\Delta T$ dependence of the subthreshold voltage amplitude for the different input configurations. For simplicity, we assumed that subthreshold membrane potential predicts ITD-induced spike rate differences well (van der Heijden et al., 2013) and that these spikes follow Poisson statistics. The relative Fisher information of the subthreshold signal was thus calculated according to the following:

$$
F(\Delta T)=\frac{f^{\prime}(\Delta T)^{2}}{f(\Delta T)}
$$

and normalized to the peak value for the single bouton simulation. As expected, information was maximum at the steepest part of the $\Delta T$ response function. Fisher information was greater for distributed synaptic input compared with a single localized synaptic site, and highest for the most distributed synapse we simulated (6 synaptic boutons). Distributed synapses could therefore be physiologically relevant features of the MSO circuit and contribute to ITD coding.

\section{Microstructure of synaptic boutons}

Our modeling of ITD sensitivity was based on the implicit assumption that there are no cycle-by-cycle variations of the synaptic conductance. This assumption requires a large number of readily releasable vesicles to continuously supply transmitter release. A large readily releasable pool of continuously available vesicles requests that there is a large number of active zones with independent release sites. To test the anatomic basis of such an assumption, we performed a $3 \mathrm{D}$ reconstruction of TEM images of a single somatodendritic element of an MSO neuron to identify active zones in single boutons. In addition, these data can also indicate whether input fibers are myelinated in the vicinity of the synaptic contacts.

We partly reconstructed an $\sim 25-\mu \mathrm{m}$-long dendritic segment close to the soma, which allowed us to identify several synaptic boutons containing vesicles and active zones (Fig. 12A). The boutons extended over several micrometers (in cross sections) and showed round vesicles indicative of glutamatergic synapses (Fig. 12B-F; but see Tao et al., 2018). Boutons generally contained multiple active zones even in the single plane visible from cross sections (Fig. 12B-F). We generally found no dark myelinated rings close to the dendrite or the synaptic boutons. Myelinated fibers were visible at some distance from the dendrite (Fig. 12A); however, it remains unclear whether these are MSO inputs or other axons passing by. To allow rough quantitative estimates, we performed a 3D reconstruction (Fig. 12G), which indicated a high density of active zones (44 on a $32 \mu \mathrm{m}^{2}$ reconstructed dendritic surface). Overlays with $3 \mathrm{D}$ bouton reconstructions suggested a lower bound of roughly five active zones per bouton on average. This lower bound is consistent with (1) electrophysiological estimates from Couchman et al. (2010), and (2) the following geometric consideration: from the cross sections (Fig. 12B-F), we estimate the contact surface of a single bouton of at least $\sim 4 \mu \mathrm{m}^{2}$ (in longitudinal sections, boutons extend over multiple micrometers; in cross sections, boutons extend over $\sim 1-2 \mu \mathrm{m})$. Further assuming that the dendritic surface is fully covered with boutons at the same density as observed in the reconstruction (Fig. 12G) amounts to a lower bound for the number of active zones per bouton of $4 \mu \mathrm{m}^{2} \cdot \frac{44}{32 \mu \mathrm{m}^{2}}=5.5$.

\section{Effect of fiber numbers on MSO function}

So far, our model simulations were only concerned with the effect of axo-dendritic organization on the processing of inputs from a single fiber. MSO neurons, however, receive multiple input fibers (see also Discussion); we thus attempted to make model-based predictions of the number of fibers targeting an MSO dendrite, given the presented anatomic constraints. Since EPSP amplitudes not only reflect the summed synaptic release from multiple release sites but also depend on the filling states of the vesicle pools in combination with the release probability, we implemented stochasticity in synaptic transmission with a 
A

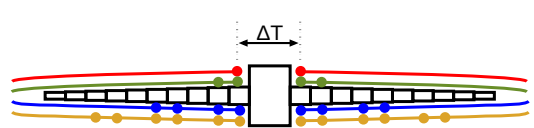

B

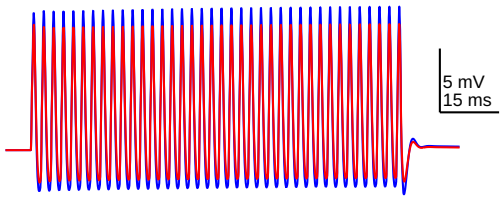

D

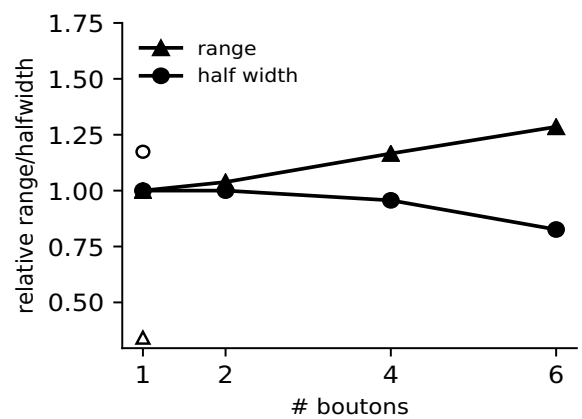

C

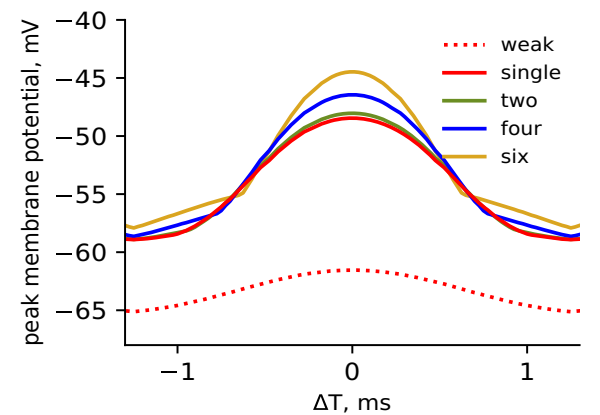

$\mathbf{E}$

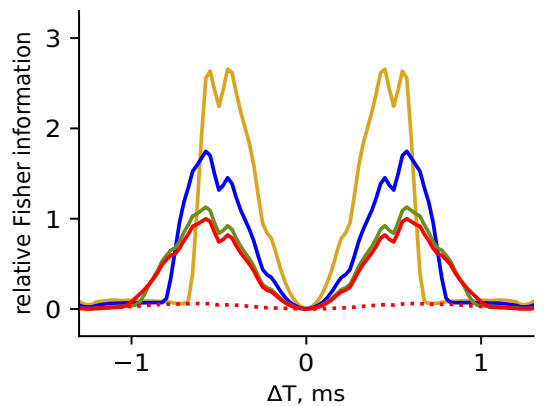

Figure 11. Distributed synapses facilitate binaural coincidence detection. $A$, Different synaptic innervation patterns, with $1,2,4$, or 6 synaptic boutons, tested with varying input timing between dendrites. $\boldsymbol{B}$, Example traces of simulations with 1 (red) or 4 (blue) synapses per axon. Total synaptic conductance $=37 \mathrm{nS}$ per dendrite; ITD $=0.4 \mathrm{~ms}$. $\boldsymbol{C}$, Peak somatic membrane potential generated during a $400 \mathrm{~Hz}$ train of EPSGS, with varying $\Delta T$. Dashed red line is derived from a single synapse with physiological average bouton conductance $37 / 4 \mathrm{nS}$. D, Response function ranges and half-widths relative to the values for a single bouton synapse. Open symbols represent results obtained with physiological average bouton conductance $37 / 4 \mathrm{nS}$. $\boldsymbol{E}$, Fisher information across the $\Delta T$ response functions for each synaptic condition. Colors correspond to $C$. Simulations were performed with an axonal conduction speed of $1 \mathrm{~m} / \mathrm{s}$.

single-pool depletion model constrained by measurements from Couchman et al. (2010). As additional, yet unconstrained, model parameter, we also took into account the temporal imprecision among fibers defined as the range of time differences between input spikes of different fibers that are elicited by the same stimulus cycle. Axonal conduction failures are not included in the model.

Assuming that, under in vivo conditions, the vesicle pool never attains its maximum filling level (Hermann et al., 2007), we restricted our analysis to a $30 \mathrm{~ms}$ time interval, $10 \mathrm{~ms}$ after the onset of a $500 \mathrm{~Hz}$ stimulus. In every cycle, the amplitudes of the synaptic conductances at a dendritic segment fluctuate, reflecting the stochasticity of the transmission model (Fig. 13A). However, since the synaptic depolarization results from the conductances of several input fibers, each having four randomly selected contact sites at the dendrite, amplitude fluctuations are already partly averaged out at the somatic membrane potential (Fig. $13 B)$. For further analysis, we split up the simulated voltage signal in oscillation cycles of the input stimulus (Fig. 13C) and derived the mean voltage peak (above rest) as a function of the time shift between dendritic stimulation as a proxy for the ITD tuning curve (Fig. 13D).

We then simulated such tuning functions for systematically varying imprecision and fiber number (Fig. 13E). While the breakdown of putative ITD tuning in our simulations clearly indicates an upper bound for the imprecision values at $\sim 400 \mu \mathrm{s}$ independent of the fiber number, the dependence of functional parameters on fiber number is gradual (Fig. 13E,F). Both maximum (at best ITD) and minimum (at worst ITD) voltage increase with fiber number as well as the coding range (maximum - minimum); however, coding range has its steepest slope between 7 and 10 fibers.
As a further experimental constraint, we consider the voltage threshold determined as $\sim 10 \mathrm{mV}$ above rest in vitro (Scott et al., 2010). Voltage maxima barely $>10 \mathrm{mV}$ can already be obtained in our simulation with four fibers; however, robust depolarization above in vitro thresholds (at best ITD) requires $\geq 7$ fibers. Particularly, taking into account additional active conductances under in vivo conditions, such as inhibition and HCN channels, probably requires even higher depolarization; thus, the range of 7-15 input fibers per dendrite seems plausible also from this perspective.

\section{Discussion}

We have shown that excitatory axons from the cochlear nucleus innervate MSO neurons with a variable but stereotyped pattern. Axons approach MSO neurons at relatively distal positions and run parallel to the dendrite, forming several synaptic boutons, before terminating with a bouton relatively close to the soma. They stay close to the dendrite and only occasionally form branches, which themselves run in parallel to the dendrite. The observed distribution of boutons along the dendrites, and the tendency of terminal boutons to be positioned close to the soma, make it unlikely that dendritic propagation delays have significant differential effects on the timing of excitatory inputs.

A similar anatomic arrangement has been proposed for the afferent fibers to octopus cells (Fekete et al., 1984), another auditory brainstem cell type with a leaky membrane. The similarity of the innervation patterns may indicate a general computational rule in dendrites of leaky cells with ultra-fast dendritic integration. Our computational model revealed that different patterns of synaptic input to the dendrites distinctly affect the somatic voltage response. The somatic membrane response to distributed 
inputs was larger and briefer, and occurred earlier, compared with the response to a spatially restricted synaptic input. Distributed inputs in the model generated larger low threshold potassium currents yielding higher temporal precision. Also, the nondepolarized dendritic compartments act as current sinks and thus simultaneous depolarization of the whole dendrite boosts EPSP amplitude. The combination of anatomic distribution of synapses and nonlinear membrane dynamics thus leads to a novel but counterintuitive effect that dendrites can speed up and amplify subthreshold synaptic input without additional recruitment of dendritic sodium or calcium channels. The resulting sharpening of EPSPs naturally improves coincidence detector precision, adding to earlier findings by (Agmon-Snir et al., 1998) that binaural stimulation generates larger EPSP amplitudes in binaural coincidence detector neurons than monaural stimulation both because of the missing current sink in the otherwise unstimulated dendrite, and a reduced saturation in driving force in bilateral versus unilateral depolarization.

We also provide complementary anatomic evidence that corroborates the physiological evidence for relatively low numbers of input fibers from Couchman et al. (2010). They determined that excitatory (as well as inhibitory) inputs to MSO neurons are large, with activation of only at most four excitatory fibers per dendrite needed to stimulate action potential generation from rest. Given that we observe a median of four boutons per axon, and up to 30 boutons per dendrite (which is definitely a gross underestimation, as not all axons were filled), MSO neurons most likely receive at least 7 input fibers and thus 2-4 times this minimum amount of excitatory input per dendrite. Since inputs to the MSO also exhibit substantial short-term synaptic depression, especially at higher frequencies (Couchman et al., 2010), these additional (i.e., beyond the minimum required) inputs maintain sufficient excitatory drive during prolonged stimuli (see Fig. 13) (Franken et al., 2014).

Our results are also in accordance with previous anatomic studies. An electron microscopy study of the cat by Lindsey (1975, his Fig. 9) showed at least one example of an axon innervating an MSO neuron with more than one bouton. Additionally, Beckius et al. (1999, their Fig. 7) provided anatomic hints of multiple synaptic boutons from a single axon innervating an MSO neuron, although they did not stain MSO neurons in that study. We demonstrate that, in the gerbil, almost all excitatory inputs form multiple contact sites along MSO dendrites.

Previous studies have shown that multiple active zones, hence release sites, can be present within a single synaptic bouton (Lindsey, 1975; Kiss and Majorossy, 1983). Excitatory axons therefore effectively form large, distributed synapses on MSO
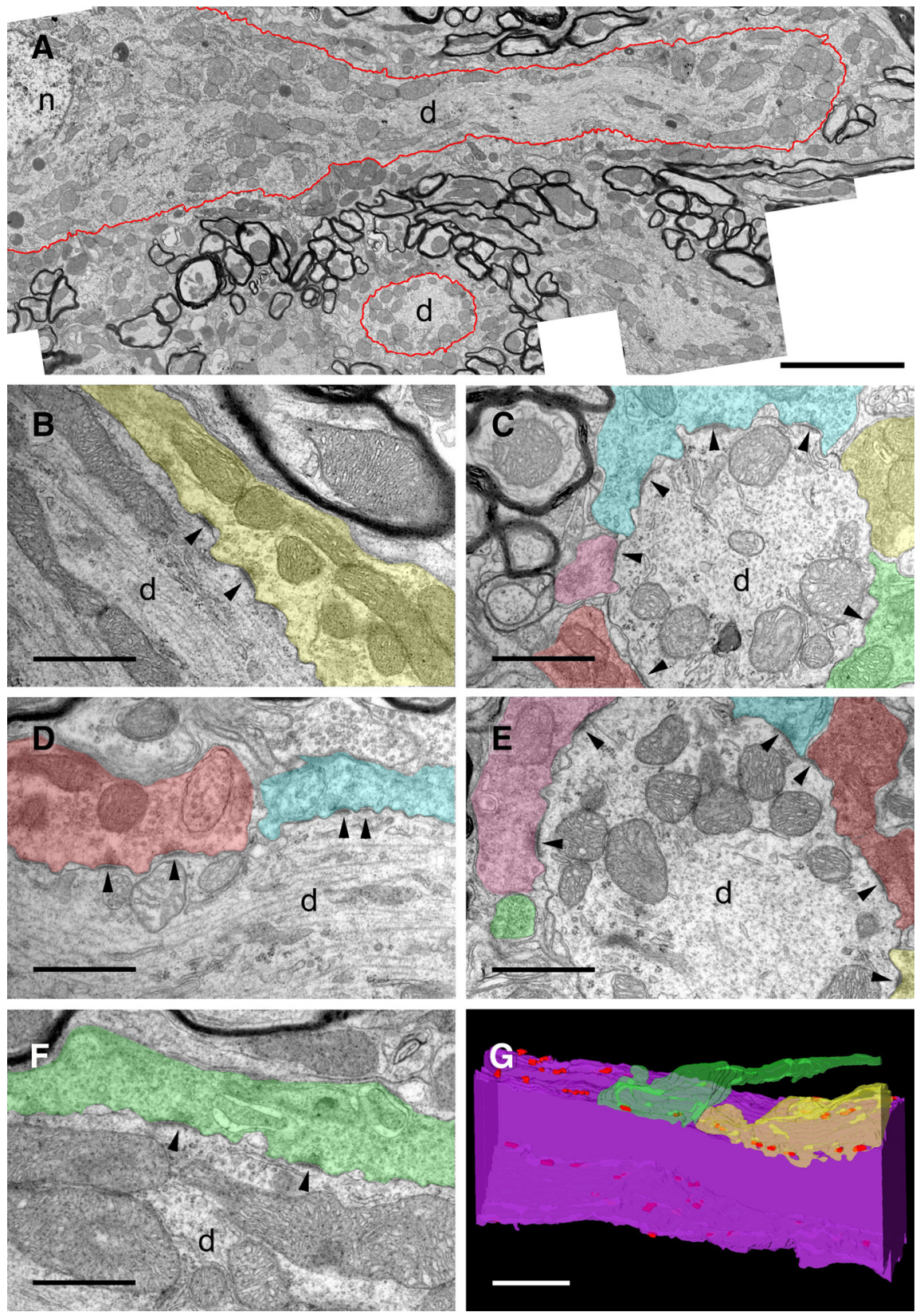

Figure 12. TEM illustrations and reconstruction of a proximal dendritic segment. $A$, Overview (11kx electron micrograph resolution) of a dendrite part (d) proximal to the cell nucleus (n) and orthogonal dendrite (d). Red line indicates borders. Scale bar, $5 \mu \mathrm{m}$. B $\boldsymbol{B}$, Examples (22kx electron micrograph resolution) of cross sections displaying presynaptic boutons (colored) with round vesicles and active zones (arrow heads). Scale bars, $1 \mu \mathrm{m}$. G, 3D reconstruction of the dendrite (surface 32 $\mu \mathrm{m}^{2}$ ) shown in $\boldsymbol{A}$, with two example boutons, including terminal axonal parts (yellow and green). Red dots indicate active zones (in total 44). Scale bar, $2 \mu \mathrm{m}$.

neurons. From our electron micrographs we estimated an average density of active zones of 1.375 per $\mu \mathrm{m}^{2}$. Thus, a typical main trunk of a $100-\mu \mathrm{m}$-long dendrite with $1 \mu \mathrm{m}$ radius would support 864 active zones, assuming the density of active zones constant along the dendrite, which is not necessarily the case. Indeed, because axons approach the dendrite from the distal end and not all make their initial synaptic contacts right at the beginning of the dendrite, it is reasonable to assume that the density of active zones per area increases toward the soma. Nevertheless, using the number of 864 active zones, we can now estimate an upper bound for the number of fibers per dendrite. Our estimate of at least 5 active zones per bouton would predict $\sim 172$ boutons 
A

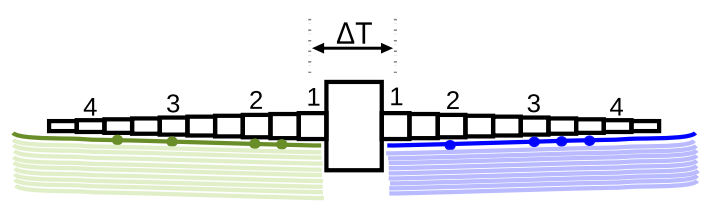

Sum synaptic conductance at dendritic compartment

$\Delta \mathrm{T}=0.5 \mathrm{~ms}$

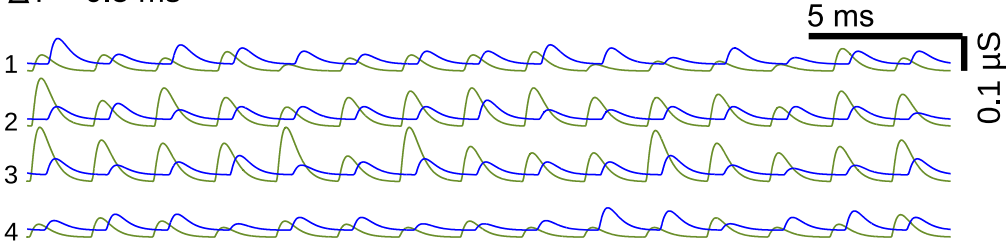

B
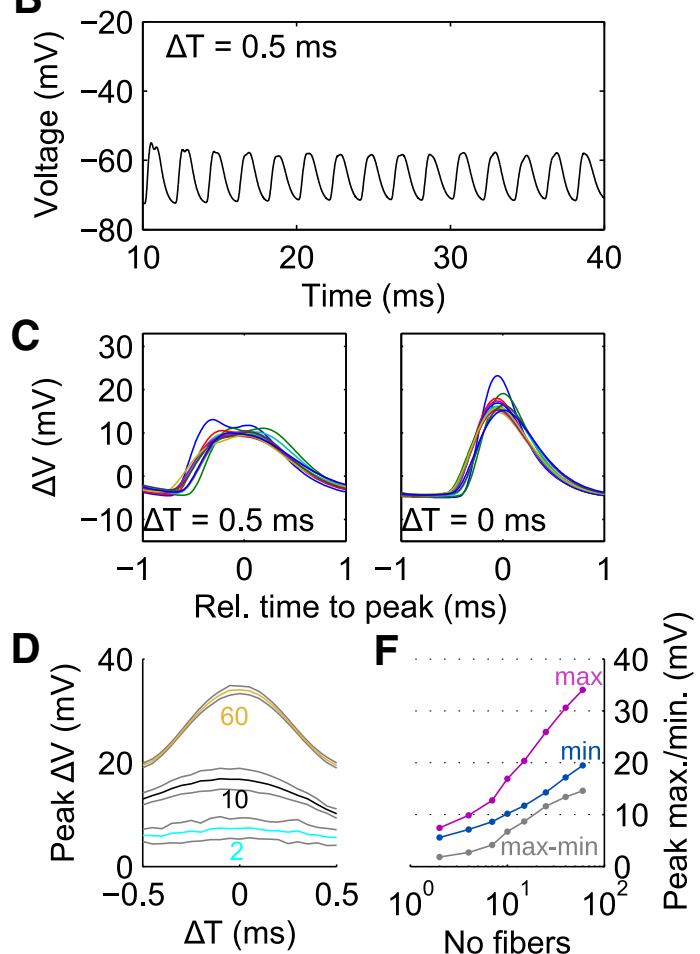

E

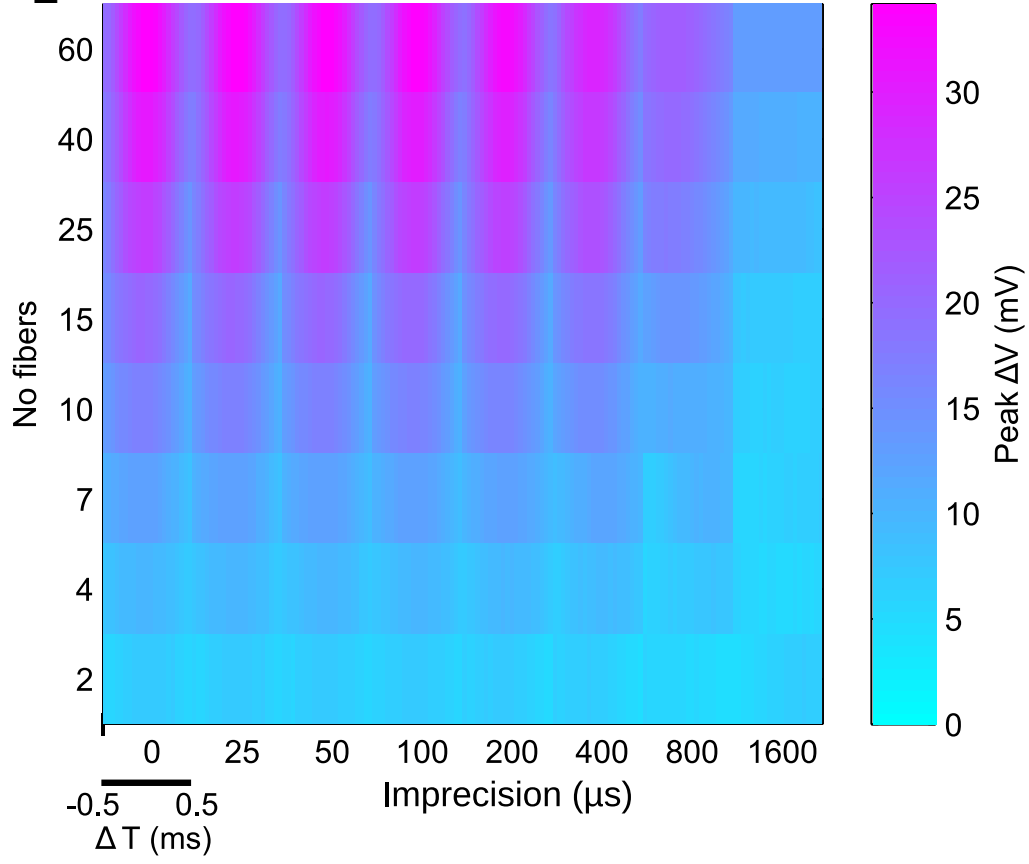

Figure 13. Model predictions on the number of input fibers. $\boldsymbol{A}$, Model design (left) and example synaptic conductance traces (right) at four exemplary dendritic sites (1-4 as indicated) for an ITD of $\Delta T=0.5 \mathrm{~ms}$ and a stimulus frequency of $500 \mathrm{~Hz}$. Green and blue represent inputs to the two dendrites. Traces are obtained for 10 fibers per dendrite and a temporal imprecision of $50 \mu \mathrm{s}$. The four randomly selected synaptic locations (discs) are only shown for the first axon; the other nine axons per dendrite are depicted in fainter color without their synaptic contacts. $\boldsymbol{B}$, Somatic voltage traces derived from inputs in $\boldsymbol{A}$. C, Overlay of voltage traces (resting potential subtracted) from all cycles aligned to zero Hilbert phase of the $5 \mathrm{~Hz}$ high-pass filtered voltage signal for $\Delta=0.5 \mathrm{~ms}$ (left) and $\Delta T=0$ (right). Each cycle is shown in a different color. $\boldsymbol{D}$, Mean voltage peak (resting potential subtracted) as a function of $\Delta T$. Colors represent different numbers of input fibers as indicated. Gray lines indicate the SEM over cycles. $\boldsymbol{E}$, Response functions as in $\boldsymbol{D}$ (colors represent peak voltages) for as a function of imprecision and the numbers of input fibers. $\boldsymbol{F}$, Maximum (purple), minimum (blue), and maximum - minimum (gray) of the response functions from $\boldsymbol{D}, \boldsymbol{E}$ as a function of fiber number for imprecision $50 \mu$ s. Simulations were performed with an axonal conduction speed of $1 \mathrm{~m} / \mathrm{s}$.

per dendrite, and thus (taking the median of four boutons per axon) amounts to 43 fibers per dendrite. A reconstruction-based estimate for the dendritic surface of $\sim 1000 \mu \mathrm{m}^{2}$ (Rautenberg et al., 2009) would increase this upper bound to $\sim 70$ fibers per side, which, however, seems exaggerated, given the model prediction from Figure 13F, which suggests that realistic voltage deflections and suitable coding range are rather in agreement with fiber numbers in the range of few tens. Independent of the estimated number of fibers, our findings definitely show that each single presynaptic spike likely triggers tens of independent synaptic transmission processes at the active zones, thereby establishing secure single-fiber transmission needed to evoke action potentials (Franken et al., 2014) despite probabilistic synaptic transmission. This is particularly important when the number of inputs is small, which also could explain the occasional appearance of isolated postsynaptic potentials in in vivo intracellular recordings (van der Heijden et al., 2013; Franken et al., 2015).
Our results from modeling excitatory inputs to single MSO neuron dendrites suggest that myelin could somewhat affect EPSP timing. Both myelinated and unmyelinated axons between two synaptic boutons were observed in Lindsey (1975, compare his Fig. 9 and Fig. 9, plate 5). Several other electron microscopy studies have also shown myelinated sections of axons immediately adjacent to synaptic boutons in cat (Clark, 1969; Kiss and Majorossy, 1983). Electron micrographs of ferret MSO show many myelinated axons adjacent to MSO dendrites (BrunsoBechtold et al., 1990). However, these studies do not show that these axons are the same as those forming synaptic boutons, nor the axon's origin. In general, a species difference or a heterogeneity of myelination patterns of input fibers cannot be ruled out so far. Our electron micrograph images show that, at least close to the terminal, axons are unmyelinated, despite myelin being clearly visible more distant to the dendrite. Whether these myelinated fibers are anteroventral cochlear nucleus fibers synapsing at other parts of the dendrite, stem from other sources, or 
have different destinations remains to be investigated by largerscale electron micrograph reconstructions. Our modeling, however, shows that axonal myelination along a short stretch parallel to the dendrite does not seem to have strong implications for postsynaptic integration.

The innervation of binaural coincidence detectors in the nucleus laminaris of barn owls shows a markedly different pattern (Carr and Boudreau, 1993). Most importantly, there the terminals of the axons from either side are not anatomically separated, but also the number of 100 input fibers per side seems substantially larger than our estimates. The pattern of excitatory innervation that we observe is therefore likely not only constrained by functional factors, but also evolutionary and developmental ones. In mammals, axons growing from the cochlear nucleus toward the MSO would naturally approach the distal ends of MSO dendrites because of the relative positions of the two nuclei. Competition from contralateral axons appears to restrict innervation to the lateral dendritic field (Kitzes et al., 1995). Repetition of our study with young animals before and after hearing could shed some light on the developmental constraints. In juvenile animals, MSO dendrites are more complex (Rautenberg et al., 2009), and the excitatory inputs are distributed in small bouton-like structures throughout the neuropil (Franzen et al., 2020). As the single-fiber size of excitatory inputs increases during late postnatal development (Franzen et al., 2020), we would postulate that bouton size, or the number of contact sites, and therefore the number of active zones, also increases during development. Moreover, this process is likely regulated by activity (Franzen et al., 2020). Our computational model using anatomic input patterns shows that such an increase in single-fiber strength physiologically subserves precise and ongoing coincidence detection.

One argument in favor of the physiological relevance of the observed input pattern is the contrast in synaptic strengths between MSO and lateral superior olive (LSO). While the MSO has fewer, strong excitatory inputs (Couchman et al., 2010), the mouse (i.e., high-frequency) LSO has been shown to have many, weaker excitatory inputs (Gjoni et al., 2018). The MSO is believed to have evolved through duplication and subsequent alteration of the LSO circuitry (Grothe and Pecka, 2014), suggesting that the pattern of large distributed synapses in the MSO is likely a specific adaptation to cycle-by-cycle ITD detection, predicting similar adaptations in low-frequency segments of the LSO.

\section{References}

Agmon-Snir H, Carr CE, Rinzel J (1998) The role of dendrites in auditory coincidence detection. Nature 393:268-272.

Baumann VJ, Lehnert S, Leibold C, Koch U (2013) Tonotopic organization of the hyperpolarization-activated current (Ih) in the mammalian medial superior olive. Front Neural Circuit 7:117.

Beckius GE, Batra R, Oliver DL (1999) Axons from anteroventral cochlear nucleus that terminate in medial superior olive of cat: observations related to delay lines. J Neurosci 19:3146-3161.

Brand A, Behrend O, Marquardt T, McAlpine D, Grothe B (2002) Precise inhibition is essential for microsecond interaural time difference coding. Nature 417:543-547.

Brunso-Bechtold JK, Henkel CK, Linville C (1990) Synaptic organization in the adult ferret medial superior olive. J Comp Neurol 294:389-398.

Carr CE, Boudreau RE (1993) Organization of the nucleus magnocellularis and the nucleus laminaris in the barn owl: encoding and measuring interaural time differences. J Comp Neurol 334:337-355.

Clark GM (1969) The ultrastructure of nerve endings in the medial superior olive of the cat. Brain Res 14:293-305.

Couchman K, Grothe B, Felmy F (2010) Medial superior olivary neurons receive surprisingly few excitatory and inhibitory inputs with balanced strength and short-term dynamics. J Neurosci 30:17111-17121.
Couchman K, Grothe B, Felmy F (2012) Functional localization of neurotransmitter receptors and synaptic inputs to mature neurons of the medial superior olive. J Neurophysiol 107:1186-1198.

Fekete DM, Rouiller EM, Liberman MC, Ryugo DK (1984) The central projections of intracellularly labeled auditory nerve fibers in cats. J Comp Neurol 229:432-450.

Franken TP, Bremen P, Joris PX (2014) Coincidence detection in the medial superior olive: mechanistic implications of an analysis of input spiking patterns. Front Neural Circuits 8:42.

Franken TP, Roberts MT, Wei L, Golding NL, Joris PX (2015) In vivo coincidence detection in mammalian sound localization generates phase delays. Nat Neurosci 18:444-452.

Franzen DL, Gleiss SA, Kellner CJ, Kladisios N, Felmy F (2020) Activity-dependent calcium signaling in neurons of the medial superior olive during late postnatal development. J Neurosci 40:1689-1700.

Gentet LJ, Stuart GJ, Clements JD (2000) Direct measurement of specific membrane capacitance in neurons. Biophys J 79:314-320.

Gjoni E, Zenke F, Bouhours B, Schneggenburger R (2018) Specific synaptic input strengths determine the computational properties of excitation-inhibition integration in a sound localization circuit. J Physiol 596:49454967.

Goldberg JM, Brown PB (1969) Response of binaural neurons of dog superior olivary complex to dichotic tonal stimuli: some physiological mechanisms of sound localization. J Neurophysiol 32:613-636.

Grothe B, Pecka M (2014) The natural history of sound localization in mammals: a story of neuronal inhibition. Front Neural Circuit 8:116.

Grothe B, Pecka M, McAlpine D (2010) Mechanisms of sound localization in mammals. Physiol Rev 90:983-1012.

Hermann J, Pecka M, von Gersdorff H, Grothe B, Klug A (2007) Synaptic transmission at the calyx of Held under in vivo like activity levels. J. Neurophysiol 98:807-820.

Joris PX, Carney LH, Smith PH, Yin TC (1994) Enhancement of neural synchronization in the anteroventral cochlear nucleus: I. Responses to tones at the characteristic frequency. J Neurophysiol 71:1022-1036.

Kapfer C, Seidl AH, Schweizer H, Grothe B (2002) Experience-dependent refinement of inhibitory inputs to auditory coincidence-detector neurons. Nat Neurosci 5:247-253.

Kiss A, Majorossy K (1983) Neuron morphology and synaptic architecture in the medial superior olivary nucleus. Exp Brain Res 52:315-327.

Kitzes LM, Kageyama GH, Semple MN, Kil J (1995) Development of ectopic projections from the ventral cochlear nucleus to the superior olivary complex induced by neonatal ablation of the contralateral cochlea. J Comp Neurol 353:341-363.

Klumpp RG, Eady HR (1956) Some measurements of interaural time difference thresholds. J Acoust Soc Am 28:859-860.

Lehnert S, Ford MC, Alexandrova O, Hellmundt F, Felmy F, Grothe B, Leibold C (2014) Action potential generation in an anatomically constrained model of medial superior olive axons. J Neurosci 34:5370-5384.

Leibold C (2010) Influence of inhibitory synaptic kinetics on the interaural time difference sensitivity in a linear model of binaural coincidence detection. J Acoust Soc Am 127:931-942.

Lindsey BG (1975) Fine structure and distribution of axon terminals from cochlear nucleus on neurons in the medial superior olivary nucleus of the cat. J Comp Neurol 160:81-103.

Mathews PJ, Jercog PE, Rinzel J, Scott LL, Golding NL (2010) Control of submillisecond synaptic timing in binaural coincidence detectors by $\mathrm{K}(\mathrm{v}) 1$ channels. Nat Neurosci 13:601-609.

Meeks JP, Mennerick S (2007) Action potential initiation and propagation in CA3 pyramidal axons. J Neurophysiol 97:3460-3472.

Myoga MH, Lehnert S, Leibold C, Felmy F, Grothe B (2014) Glycinergic inhibition tunes coincidence detection in the auditory brainstem. Nat Commun 5:3790.

Nabel AL, Callan AR, Gleiss SA, Kladisios N, Leibold C, Felmy F (2019) Distinct distribution patterns of potassium channel sub-units in somatodendritic compartments of neurons of the medial superior olive. Front Cell Neurosci 13:38.

Pecka M, Brand A, Behrend O, Grothe B (2008) Interaural time difference processing in the mammalian medial superior olive: the role of glycinergic inhibition. J Neurosci 28:6914-6925.

Rautenberg PL, Grothe B, Felmy F (2009) Quantification of the three-dimensional morphology of coincidence detector neurons in the medial 
superior olive of gerbils during late postnatal development. J Comp Neurol 517:385-396.

Scott LL, Mathews PJ, Golding NL (2010) Perisomatic voltage-gated sodium channels actively maintain linear synaptic integration in principal neurons of the medial superior olive. J Neurosci 30:2039-2050.

Seidl AH, Rubel EW (2016) Systematic and differential myelination of axon collaterals in the mammalian auditory brainstem. Glia 64:487-494.

Sinclair JL, Fischl MJ, Alexandrova O, $\mathrm{He} \beta$ M, Grothe B, Leibold C, KoppScheinpflug C (2017) Sound-evoked activity influences myelination of brainstem axons in the trapezoid body. J Neurosci 37:8239-8255.

Smith PH, Joris PX, Yin TC (1993) Projections of physiologically characterized spherical bushy cell axons from the cochlear nucleus of the cat: evidence for delay lines to the medial superior olive. J Comp Neurol 331:245-260.

Stotler WA (1953) An experimental study of the cells and connections of the superior olivary complex of the cat. J Comp Neurol 98:401-431.

Tao CL, Liu YT, Sun R, Zhang B, Qi L, Shivakoti S, Tian CL, Zhang P, Lau PM, Zhou ZH, Bi GQ (2018) Differentiation and characterization of excitatory and inhibitory synapses by cryo-electron tomography and correlative microscopy. J Neurosci 38:1493-1510. van der Heijden M, Lorteije JA, Plauška A, Roberts MT, Golding NL, Borst JG (2013) Directional hearing by linear summation of binaural inputs at the medial superior olive. Neuron 78:936-948.

Weidner C, Schmelz M, Schmidt R, Hansson B, Handwerker HO, Torebjörk HE (1999) Functional attributes discriminating mechano-insensitive and mechano-responsive C nociceptors in human skin. J Neurosci 19:1018410190.

Werthat F, Alexandrova O, Grothe B, Koch U (2008) Experience-dependent refinement of the inhibitory axons projecting to the medial superior olive. Dev Neurobiol 68:1454-1462.

Winters BD, Jin SX, Ledford KR, Golding NL (2017) Amplitude normalization of dendritic EPSPs at the soma of binaural coincidence detector neurons of the medial superior olive. J Neurosci 37:3138-3149.

Yin TC, Chan JC (1990) Interaural time sensitivity in medial superior olive of cat. J Neurophysiol 64:465-488.

Zhou YY, Carney LH, Colburn HS (2005) A model for interaural time difference sensitivity in the medial superior olive: interaction of excitatory and inhibitory synaptic inputs, channel dynamics, and cellular morphology. J Neurosci 25:3046-3058 